\title{
Relativistic Invariance and Charge Conjugation in Quantum Field Theory
}

\author{
Daniele Guido * and Roberto Longo * \\ Dipartimento di Matematica, Università di Roma “Tor Vergata", \\ Via della Ricerca Scientifica, I-00133 Roma, Italy
}

Received January 13, 1992; in revised form March 27, 1992

\begin{abstract}
We prove that superselection sectors with finite statistics in the sense of Doplicher, Haag, and Roberts are automatically Poincaré covariant under natural conditions (e.g. split property for space-like cones and duality for contractible causally complete regions). The same holds for topological charges, namely sectors localized in space-like cones, providing a converse to a theorem of Buchholz and Fredenhagen. We introduce the notion of weak conjugate sector that turns out to be equivalent to the DHR conjugate in finite statistics. The weak conjugate sector is given by an explicit formula that relates it to the PCT symmetry in a Wightman theory. Every Euclidean covariant sector (possibly with infinite statistics) has a weak conjugate sector and the converse is true under the above natural conditions. On the same basis, translation covariance is equivalent to the property that sectors are sheaf maps modulo inner automorphisms, for a certain sheaf structure given by the local algebras. The construction of the weak conjugate sector also applies to the case of local algebras on $S^{1}$ in conformal theories. Our main tools are the Bisognano-Wichmann description of the modular structure of the von Neumann algebras associated with wedge regions in the vacuum sector and the relation between Jones index theory for subfactors and the statistics of superselection sectors.
\end{abstract}

\section{Introduction}

According to the Haag-Kastler approach to Quantum Field Theory [19], the physical content of the theory is encoded in the net of von Neumann algebras $\mathscr{A}(\mathcal{O})$ of local observables associated with double cones of the Minkowski space. In this framework the physical representations (the superselection sectors or quantum charges introduced in [38]) are to correspond to a family of (unitary equivalence class of) representations of the quasi-local $C^{*}$-algebra $\mathscr{A}=\bigcup \mathscr{A}(\mathcal{O})^{-}$. Since not all

* Supported in part by Ministero della Ricerca Scientifica and CNR-GNAFA E-mail GUIDO@, MAT.UTOVRM.IT, LONGO@MAT.UTOVRM.IT 
representations of $\mathscr{A}$ (as a $C^{*}$-algebra) have a physical meaning, criteria are needed to select the physically relevant representations.

The action of the Poincare group on the Minkowski space give rise to an action on $\mathscr{A}$. There is a distinguished representation, the vacuum sector, where this action is unitarily implemented with positive energy, i.e. the spectrum of the unitary action of the translation subgroup is contained in the forward light cone.

As a selection criterium, Borchers [3] proposed to consider the class of representations of $\mathscr{A}$ that are Poincare covariant with positive energy.

Depending on the principle of locality and with the aim of describing short range interactions Doplicher, Haag, and Roberts (DHR) [14] considered the class of localized representations, namely representations that become equivalent to the vacuum representation if both are restricted to the $C^{*}$-algebra $\mathscr{A}\left(\mathcal{O}^{\prime}\right)$ of the spacelike complement of a double cone $\mathcal{O}$. They defined also a parameter, called statistical dimension, associated with such representations, and restricted their attention to the finite statistics case. In particular, a suitably defined conjugate charge exists within this class.

Buchholz and Fredenhagen [7] observed that wider localization regions are necessary in order to describe general massive representations (representations where the spectrum of the translation subgroup contains an isolated mass shell), in particular, topological charges. Indeed, they proved that any massive representation obeying the Borchers criterium can be localized in a space-like cone and has finite statistics [16].

Thus Poincaré covariance and localizability give related criteria to select physical representations. But the problem of whether localizable representations with finite statistics are automatically Poincare covariant is a long-standing problem since the DHR work [15].

A main result in this paper is that under general hypotheses ${ }^{1}$ any sector with finite statistics and localizable in a space-like cone is indeed Poincaré covariant, with positive energy. Hence, in the massive case, Borchers condition selects the same class of sectors as the Buchholz-Fredenhagen localization together with finite statistics.

Moreover, we shall introduce a notion of weak conjugate sector that becomes equivalent to the DHR conjugation in the finite statistics case, and show that a weak conjugate charge exists iff $\varrho$ is covariant under the Euclidean group.

Our results are obtained by analyzing the relations among fundamental properties of a sector localizable in a space-like cone, as it will be illustrated below. We anticipate these relations in the following summarizing diagram:

Finite statistics

$\uparrow$

$\exists$ DHR conjugate

$\Rightarrow \quad$ Poincaré covariance

$\Downarrow$

$\exists$ weak conjugate

$\Downarrow$

$\Leftrightarrow$ Euclidean covariance

$\Downarrow$

$\Downarrow$

$\varrho$ is a sheaf map modulo inners $\Leftrightarrow$ Translation covariance.

\footnotetext{
1 For example, we may assume the split property for space-like cones and the duality property
} for contractible causally complete regions (see Sect. 6) 
Before a more detailed discussion of the content of this paper, we mention that the original motivation for our analysis was the purpose of a better understanding of the consequences of the equation

$$
\operatorname{Ind}(\varrho)=d(\varrho)^{2}
$$

found in [26]. Here the sector $\varrho$ is viewed as an endomorphism of $\mathscr{A}$ and the formula relates its index [24] with the square of the statistical dimension [14] of $\varrho$. Most of this paper relies on some ideas and results on index theory in infinite factors [27]. The essential mathematical structure is explained in Sect. 2 and in the Appendix.

Poincaré covariance, the particle-antiparticle symmetry and the notion of weak conjugate. In the Wightman approach to quantum field theory the particleantiparticle symmetry is expressed by the well known PCT theorem; it gives an antiunitary operator that implements the product of space reflection, time inversion and charge conjugation. In the DHR setting [14], with space-time dimension greater than 2 , sectors are represented by endomorphisms of $\mathscr{A}$ (modulo inners) and any sector $\varrho$ with finite statistics has a conjugate sector $\bar{\varrho}$, characterized by the property that $\varrho \circ \varrho$ contains the identity, namely

$$
\exists v \in \mathscr{A}, \quad v \neq 0: \quad \varrho \circ \varrho(x) v=v x, \quad x \in \mathscr{A} .
$$

Now, according with a theorem of Bisognano and Wichmann [2], the PCT antiunitary is the product of the modular conjugation [37] of the von Neumann algebra $\mathscr{R}(W)$ associated to a wedge region with a spatial rotation. One may argue by this point that modular theory of von Neumann algebras and DHR charge conjugation are to be related.

This is in fact true [27]. If $M$ is an infinite factor the semiring $\operatorname{Sect}(M)$, the quotient of the endomorphisms $\operatorname{End}(M)$ of $M$ modulo inner automorphisms, has a conjugation

$$
\bar{\varrho}=\varrho^{-1} \circ \gamma_{\varrho}, \quad \varrho \in \operatorname{End}(M),
$$

where $\gamma_{\varrho}: M \rightarrow \varrho(M)$ is a canonical endomorphism [28] relating the modular structure of $M$ and $\varrho(M)$. If $\varrho$ is irreducible with finite index $\varrho$ is characterized by the analogue of the property (1.1).

Now let $\varrho$ be a morphism of $\mathscr{A}$ localized in a space-like cone $\mathscr{S}$. By essential duality, for any wedge region $W$ that contains $\mathscr{S}, \varrho$ gives rise to an endomorphism $\varrho_{W}$ of $\mathscr{R}(W)$, the weak closure of $\mathscr{A}(W)$. Therefore, we may try to construct a consistent family of conjugate endomorphisms by the formula (1.2): for each pair of wedge regions $W_{1}, W_{2}$ containing $\mathscr{S}$, we ask for a choice of $\bar{\varrho}_{W_{1}}, \bar{\varrho}_{W_{2}}$ (we have the freedom to perturb by inner automorphisms) such that

$$
\left.\bar{\varrho}_{W_{1}}\right|_{\mathscr{R}\left(W_{1} \cap W_{2}\right)}=\left.\bar{\varrho}_{W_{2}}\right|_{\mathscr{R}\left(W_{1} \cap W_{2}\right)} \text {. }
$$

If this is possible, the family of endomorphisms $\varrho_{W}$, as $W$ varies, determines a morphism $\varrho$ of $\mathscr{A}$ that we call a weak conjugate of $\varrho$. Depending on the geometrical meaning of the modular objects of the algebra of a wedge region, we will prove that the existence of a weak conjugate is equivalent to the Euclidean covariance of $\varrho$. Indeed, we shall obtain the global formula

$$
\bar{\varrho}=j \circ \varrho \circ j,
$$

where $j$ is the antiautomorphism of $\mathscr{B}(\mathscr{H})$ implemented by the PCT antiunitary, 
thus relating the charge conjugation in the Wightman formulation with the DHR conjugation of a sector in the local algebras formulation.

In finite statistics, weak conjugates and DHR conjugates coincide and we therefore deduce the Euclidean covariance. The covariance with respect to the full Poincaré group is obtained (in finite statistics and arbitrary space-time dimension) by the Bisognano-Wichmann theorem and the Connes Radon-Nikodym theorem for the modular group [11].

By means of a recent result of Borchers [4] on the Bisognano and Wichmann theorem in the algebraic setting, we will prove in Sect. 10 that finite statistics implies translation covariance within the Haag-Kastler framework.

The sheaf of local algebras. Roberts [33] has long proposed to consider a sheaf structure arising from the net of local von Neumann algebras, that clarifies the mathematical structure of the superselection sectors.

In this paper we are naturally led to consider a related but different sheaf structure. First of all with each open set $\mathcal{O}$ we associate the usual local von Neumann algebra $\mathscr{R}(\mathcal{O})$ defined by additivity. Then, to get a presheaf, one has to assign restriction maps, namely (homo-)morphisms

$$
\gamma_{\tilde{\mathcal{O}}, \mathcal{O}}: \mathscr{R}(\widetilde{\mathcal{O}}) \rightarrow \mathscr{R}(\mathcal{O})
$$

of $\mathscr{R}(\widetilde{\mathcal{O}})$ into $\mathscr{R}(\mathcal{O})$ for any open sets $\mathcal{O} \subset \widetilde{\mathcal{O}}$ with the properties

$$
\begin{gathered}
\gamma_{\mathcal{O}, \mathcal{O}}=\mathrm{id}, \\
\gamma_{\mathcal{O}_{3}, \mathcal{O}_{1}}=\gamma_{\mathcal{O}_{2}, \mathcal{O}_{1}} \circ \gamma_{\mathcal{O}_{3}, \mathcal{O}_{2}}, \quad \mathcal{O}_{1} \subset \mathcal{O}_{2} \subset \mathcal{O}_{3} .
\end{gathered}
$$

One obtains a sheaf if, furthermore, the following holds: if $\mathcal{O}=\bigcup_{i} \mathcal{O}_{i}$ and we pick an $x_{i}$ in each $\mathscr{R}\left(\mathcal{O}_{i}\right)$ with the property that $x_{i}$ and $x_{j}$ have the same restriction to $\mathcal{O}_{i} \cap \mathcal{O}_{j}$, there exists a unique $x \in \mathscr{R}(\mathcal{O})$ with $x_{i}=\gamma_{\mathcal{O}, \mathcal{O}_{i}}(x)$.

Our first observation is that if $\gamma_{\tilde{\mathcal{O}}, \mathcal{O}}$ is the canonical endomorphism of $\mathscr{R}(\widetilde{\mathcal{O}})$ into $\mathscr{R}(\mathcal{O})$ with respect to the vacuum vector (where $\mathcal{O} \subset \widetilde{\mathcal{O}}$ are bounded non-empty) then the $\mathscr{R}(\mathcal{O})$ 's form indeed a sheaf.

Now observe that the canonical endomorphisms $\gamma_{\tilde{\mathcal{O}, \mathcal{O}}}$ have a geometrical meaning for particular regions [25]. For example, if $\tilde{W} \supset W$ are wedge regions, as a corollary of the Bisognano-Wichmann theorem, $\gamma_{\tilde{W}, W}$ is a space-time translation on $\mathscr{R}(\tilde{W})$.

If $\varrho$ is an endomorphism of $\mathscr{A}$ localized in $\mathcal{O}$, the translation covariance becomes the property

$$
\varrho \circ \gamma_{\tilde{w}, W}=\operatorname{ad}(u) \circ \gamma_{\tilde{w}, W} \circ \varrho
$$

for some unitary $u \in \mathscr{R}(W)$, namely $\varrho$ is a sheaf map modulo inners on the wedges containing $\mathcal{O}$.

Furthermore, if we suppose $\varrho$ to have finite statistics, we obtain property (1.3) not only for wedges, but also for double cones containing $\mathcal{O}$.

This "geometrical" formalism appears as the natural language for the analysis of theories on topologically non-trivial manifolds, possibly arising from curved space-times. For example, conformal theories on $S^{1}$ give sheaves (and cosheaves), but not nets, of local algebras (pre-compact contractible open sets do not form a direct set in $S^{1}$ ).

According to these ideas we provide a construction of the conjugate sector in this case, where the existing method (shifting the charge at infinity) 'fails. Our 
method is to define a conjugation on Roberts cohomology classes [34], which are in one-to-one correspondence with sectors, and this will be done by providing a conjugation on cocycles. The construction is also related to the universal algebra of Fredenhagen [17], that we regard from a cosheaf point of view. It works for covariant sectors, possibly with infinite statistics, a case that we expect to be important. In Sect. 11 we shall give a first insight to this case, so far not envisaged.

This paper is an expanded version of an unpublished manuscript of the second named author.

\section{Mathematical Preliminaries and Abstract Covariance}

In this section we put in a convenient form and develop certain results on endomorphisms of factors; we refer to [27, 31] for more on this matter.

Most of this paper is based on Lemma 2.1 here. However, we also present abstract results that clarify the role of the hypotheses and have their own interest.

Let $M$ be a properly infinite von Neumann algebra, that we assume for simplicity to have separable predual. Recall that if $N$ is a properly infinite von Neumann subalgebra, the canonical endomorphism $\gamma: M \rightarrow N$ is defined by

$$
\gamma(x)=\Gamma x \Gamma^{*},
$$

where $\Gamma=J_{N} J_{M}$ is the product of a choice of the modular conjugations in the standard representation space of $M$. The endomorphism $\gamma$ is defined up to inner automorphisms of $N$.

Denote by $\operatorname{End}(M)$ the family of unital injective normal endomorphisms of $M$. If $\varrho \in \operatorname{End}(M)$ then a conjugate $\varrho$ of $\varrho$ is the endomorphism given by

$$
\bar{\varrho}=\varrho^{-1} \circ \gamma_{\varrho},
$$

where $\gamma_{\varrho}: M \rightarrow \varrho(M)$ is a canonical endomorphism [25]; $\varrho$ is unique modulo inner automorphisms of $M$, i.e. it gives a well defined conjugation in the semiring $\operatorname{Sect}(M)$, the quotient of $\operatorname{End}(M)$ with respect to inner automorphisms. If $M$ is a factor and $\varrho$ is irreducible [i.e. $\left.\varrho(M)^{\prime} \cap M=\mathbb{C} 1\right]$ with finite index (see $\left.[24,22,26]\right), \bar{\varrho}$ is characterized (modulo inners) by the existence of a non-zero intertwiner $v \in M$ of $\varrho \circ \varrho$ with the identity ([27], see also [23]):

$$
\varrho \circ \varrho(x) v=v x, \quad x \in M .
$$

Lemma 2.1. Let $M$ be a properly infinite von Neumann algebra in a standard form and $\varrho \in \operatorname{End}(M)$. If $U$ is a unitary that implements $\varrho$

$$
U x U^{*}=\varrho(x), \quad x \in M,
$$

then $\bar{U} \equiv J U^{*} J$ implements a conjugate $\bar{\varrho}$ of $\varrho$

$$
\bar{U} x \bar{U}^{*}=\bar{\varrho}(x), \quad x \in M,
$$

where $J$ is the modular conjugation of $M$.

Every conjugate endomorphism arises in this way.

Proof. The canonical endomorphism $\gamma_{\ell}: M \rightarrow \varrho(M)$ is implemented by the unitary $\Gamma_{\varrho}=J_{\varrho} J$, where $J_{\varrho}$ is a modular conjugation of $\varrho(M)$. We may choose $J_{\varrho}=U J U^{*}$, 
therefore

$$
\Gamma_{\varrho}=J_{\varrho} J=U J U^{*} J
$$

and $\bar{\varrho}=\varrho^{-1} \circ \gamma_{\varrho}$ is implemented by $J U^{*} J$.

If $V$ is a unitary, $V$ implements $\varrho$ iff $V=U v$ for some unitary $v \in M^{\prime}$, i.e. iff $\bar{V}=w \bar{U}$ for some unitary $w \in M$, iff $\bar{V}$ implements an endomorphism of $M$ whose class in $\operatorname{Sect}(M)$ coincides with the class $[\bar{\varrho}]$ of $\bar{\varrho}$.

Let now $N \subset M$ be an inclusion of infinite factors and $\varrho \in \operatorname{End}(M)$ such that $\varrho(N) \subset N$. We shall say that an endomorphism $\varrho \in \operatorname{End}(M)$ is a coherent conjugate of $\varrho$ (with respect to $N$ ) if $\varrho$ is a conjugate of $M$ with $\varrho(N) \subset N$ and $\left.\varrho\right|_{N}$ is a conjugate of $\left.\varrho\right|_{N}$.

We choose the modular conjugations of $M, N$ and denote them as usual by $J_{M}, J_{N}$. We denote by $M_{1}=J_{M} N^{\prime} J_{M}$ the extension of $N \subset M$.

Theorem 2.2. Let $N \subset M$ be an inclusion of infinite factors and $\gamma: M \rightarrow N$ a canonical endomorphism, and let $\varrho \in \operatorname{End}(M)$ with $\varrho(N) \subset N$.

The following are equivalent:

(i) There exists a coherent conjugate $\bar{\varrho}$ of $\varrho$.

(ii) $\varrho$ commutes with $\gamma$ modulo inner automorphisms of $N$, namely

$$
\gamma \circ \varrho(x)=u \varrho \circ \gamma(x) u^{*}, \quad x \in M,
$$

for some unitary $u \in N$.

(iii) There is a choice of the canonical endomorphism $\gamma: M \rightarrow N$ that restricts to a canonical endomorphism of $\varrho(M)$ in $\varrho(N)$.

(iv) There is a choice of the canonical endomorphism $\gamma_{\varrho}: M \rightarrow \varrho(M)$ that restricts to a canonical endomorphism of $N$ in $\varrho(N)$.

(v) $\varrho$ extends to an endomorphism $\tilde{\varrho}$ of $M_{1}$ in the same class of $\gamma^{-1} \circ \varrho \circ \gamma$ in $\operatorname{Sect}\left(M_{1}\right)$.

Proof. (i) $\Leftrightarrow$ (ii): Fix $U$ a unitary implementing $\varrho$ so that $\bar{U}=J_{M} U^{*} J_{M}$ implements a conjugate $\varrho$ of $\varrho$. Then (i) holds iff there exists a unitary $v \in M$ such that

$$
J_{M} U^{*} J_{M} x J_{M} U J_{M}=v J_{N} U^{*} J_{N} x J_{N} U J_{N} v^{*}
$$

for all $x \in N$, as follows by the uniqueness modulo inners of the conjugate. Equivalently,

$$
v^{\prime} \equiv J_{M} U J_{M} v J_{N} U^{*} J_{N} \in N^{\prime}
$$

or

$$
u \equiv j_{N}\left(v^{\prime}\right)=\Gamma U j_{M}(v) \Gamma^{*} U^{*} \in N,
$$

where $\Gamma=J_{N} J_{M}$ and $j_{M}(\cdot)=J_{M} \cdot J_{M}$ and so on.

Again this is equivalent to

$$
\left.\operatorname{ad}(\Gamma U)\right|_{M}=\left.\operatorname{ad}(u) \circ \operatorname{ad}(U \Gamma)\right|_{M},
$$

namely

$$
\gamma \circ \varrho(x)=u \varrho \circ \gamma(x) u^{*}, \quad x \in M .
$$

(ii) $\Leftrightarrow$ (iii): Setting $y=\varrho(x)$ in (2.1) we obtain

$$
u^{*} \gamma(y) u=\varrho \circ \gamma \circ \varrho^{-1}(y), \quad y \in \varrho(M) .
$$


This shows that $\operatorname{ad}\left(u^{*}\right) \gamma$ is a canonical endomorphism of $\varrho(M)$ in $\varrho(N)$, thus we obtain (iii). On the other hand, formula (2.5) is equivalent to (2.1), thus (iii) $\Rightarrow$ (ii). (i) $\Leftrightarrow$ (iv): $\varrho$ is a conjugate of $\varrho$ iff

$$
\gamma_{\varrho}=\varrho \circ \bar{\varrho},
$$

therefore, if $\varrho$ is a coherent conjugate of $\varrho$, then $\left.\gamma_{\varrho}\right|_{N}$ is a canonical endomorphism of $N$ into $\varrho(N)$. Conversely, if the latter holds, then $\varrho$ is a coherent conjugate by (2.6). (i) $\Leftrightarrow$ (v): By Lemma 2.1, in the equivalence (i) $\Leftrightarrow$ (ii) we may choose $U$ so that $v \in N$, therefore, (2.4) becomes

$$
\left.\operatorname{ad}(\Gamma U)\right|_{M_{1}}=\left.\operatorname{ad}(u) \circ \operatorname{ad}(U \Gamma)\right|_{M_{1}},
$$

namely

$$
\varrho(y) \equiv \operatorname{ad} U(y)=\operatorname{ad}(w) \circ \gamma^{-1} \circ \varrho \circ \gamma(y)
$$

for all $y \in M_{1}$ with $w \equiv \gamma^{-1}(u) \in M_{1}$, because $\gamma\left(M_{1}\right)=N$.

The next position should be compared with the notion of a commuting square of finite factors [32].

Proposition 2.3. Let

$$
\begin{array}{ccc}
N & \subset & M \\
\cup & & \cup \\
N_{0} & \subset & M_{0}
\end{array}
$$

be a square of inclusions of infinite factors and $\varepsilon: M \rightarrow M_{0}$ a normal faithful conditional expectation with $\varepsilon(N)=N_{0}$. Suppose that $N_{0} \subset N$ and $M_{0} \subset M$ are irreducible inclusions with the same finite index

$$
\operatorname{Ind}\left(N_{0}, N\right)=\operatorname{Ind}\left(M_{0}, M\right) .
$$

Then there exists a canonical endomorphism $\gamma: M \rightarrow N$ whose restriction to $M_{0}$ is a canonical endomorphism of $M_{0}$ into $N_{0}$.

Proof. We assume for simplicity that $M$ acts on a separable Hilbert space. Let $\varphi$ be a bicyclic state for $N_{0} \subset M_{0}$, namely $\varphi$ is a normal faithful state of $M_{0}$ represented by a jointly cyclic and separating vector for $N_{0}$ and $M_{0}$ in the GNS representation.

Then $\tilde{\varphi} \equiv \varphi \circ \varepsilon$ is a faithful state of $M$ that we represent by a cyclic and separating vector $\xi$.

Then

$$
e=\left[N_{0} \xi\right]=\left[M_{0} \xi\right] \in M_{0}^{\prime}
$$

is a Jones projection for $M_{0} \subset M$ and we let $M_{1}=\langle M, e\rangle$ be the extension of $M_{0} \subset M$. Denote by

$$
\tilde{N}_{1}=\langle N, e\rangle
$$

the von Neumann algebra generated by $N$ and $e$; if $\xi$ is cyclic for $N$, then $\tilde{N}_{1}$ is the extension $N_{1}$ of $N_{0} \subset N$.

In general, there is a normal homomorphism $\Phi$ of $\tilde{N}_{1}$ onto $N_{1}$ mapping $N_{0}$ onto $N_{0}, N$ onto $N$ and $e$ in the Jones projection $\bar{e}$ of $N_{0} \subset N$ (this may be checked directly by definitions). 
The dual expectation $\varepsilon^{\prime}: M_{1} \rightarrow M$ is characterized by

$$
\varepsilon^{\prime}(e)=\lambda \equiv \operatorname{Ind}\left(M_{0}, M\right)^{-1}
$$

while the dual expectation $\bar{\varepsilon}: N_{1} \rightarrow N$ is characterized by

$$
\bar{\varepsilon}(\bar{e})=\lambda\left(=\operatorname{Ind}\left(N_{0}, N\right)^{-1}\right),
$$

hence

$$
\bar{\varepsilon} \cdot \Phi=\Phi \cdot \varepsilon^{\prime}
$$

This implies that $\Phi$ is faithful since

$$
\begin{gathered}
z \in N_{1}^{+}, \quad \Phi(z)=0 \Rightarrow \Phi(\varepsilon(z))=0 \\
\Rightarrow \varepsilon(z)=0 \Rightarrow z=0
\end{gathered}
$$

because $\Phi \mid N$ is faithful as $N$ is a factor.

We thus conclude that $e$ is a Jones projection for $N_{0} \subset N$, too. We apply now [26] and write the operator $\Gamma=J_{M_{0}} J_{M}$ as

$$
\Gamma=V^{*} W,
$$

where $V \in M_{0}^{\prime}\left(\subset N_{0}^{\prime}\right)$ and $W \in N_{1}\left(\subset M_{1}\right)$ are isometries with final projection $e$. This also represents the operator $J_{N_{0}} J_{N}$ for suitable choice of the modular conjugations.

Corollary 2.4. Let $N \subset M$ be infinite factors and $\varrho \in \operatorname{End}(M)$ an irreducible endomorphism with finite index. Suppose that $\varrho(N) \subset N, \phi(N) \subset N$, where $\phi$ is the left inverse of $\varrho$, and $\left.\varrho\right|_{N}$ is irreducible with

$$
\operatorname{Ind}(\varrho)=\operatorname{Ind}\left(\left.\varrho\right|_{N}\right) \text {. }
$$

Then there exists a coherent conjugate endomorphism $\varrho$ of $\varrho$ with respect to $N \subset M$.

Proof. Immediate by Theorem 2.2 and Proposition 2.3.

\section{Covariance and Conjugates}

In this section we start with a Euclidean covariant sector and derive some consequences, mainly the existence of a (weak) conjugate sector. Previous constructions are contained in $[15,16,18]$. Our construction is new from several points of view. First of all neither finite statistics nor positivity of the energy assumptions are needed here. Moreover, we have an explicit set up for a conjugate morphism that will give the explicit formula of Sect. 4. Finally, under some regularity assumption, most of the arguments may be reversed, and we shall show in the next sections that covariance and existence of a conjugate are equivalent properties.

We briefly recall the main standard assumptions and definitions we will use in the following.

In $\mathbb{R}^{d}$ with Minkowski structure we consider double cones, i.e. all Poincaré transformations of the regions

$$
\left\{\left(a_{0}, \mathbf{a}\right) \in \mathbb{R}^{d} /\left|a_{0}\right|+|\mathbf{a}|<r\right\}, \quad r>0,
$$


wedges, i.e. all Poincaré transformations of the region

$$
\left\{\left(a_{0}, \mathbf{a}\right) \in \mathbb{R}^{d} /\left|a_{0}\right|<a_{1}\right\},
$$

and space-like cones, i.e. cones generated by a double cone and a point in the casual complement of its closure.

Then we will consider a net of $C^{*}$-algebras $\mathscr{A}(\mathcal{O})$ associated with double cones with locality and isotony properties, and define the algebra of quasilocal observables $\mathscr{A}$ as the inductive $C^{*}$-limit of all local algebras $\mathscr{A}(\mathcal{O})$. We define the algebra $\mathscr{A}(\mathscr{C})$ associated with a more general region $\mathscr{C}$ as the $C^{*}$-algebra generated by the algebras $\mathscr{A}(\mathcal{O})$ with $\mathscr{C} \supset \mathcal{O}$.

Poincaré transformations of $\mathbb{R}^{n}$ induce an action of the Poincaré group on the $C^{*}$-algebra $\mathscr{A}$, and there is a unique invariant state, the vacuum state. Positive energy(-momentum) in the vacuum representation $\pi_{0}$ is also assumed. We may omit the symbol $\pi_{0}$ in the following. We will write $\mathscr{R}(\mathscr{C})$ for the weak closure in the vacuum representation of the algebra $\mathscr{A}(\mathscr{C})$. If $\mathcal{O}$ is a double cone we may and do assume $\mathscr{A}(\mathcal{O})$ to be weakly closed, namely $\mathscr{A}(\mathcal{O})=\mathscr{R}(\mathcal{O})$. We recall that with this hypothesis von Neumann algebras associated to wedge regions are factors of type $I I I_{1}$ (and injective if the split property holds), see [30].

Moreover, we shall need essential duality, i.e.

$$
\mathscr{R}(W)^{\prime}=\mathscr{R}\left(W^{\prime}\right)
$$

for each wedge $W$, and the identification of the modular conjugation of $\mathscr{R}(W)$ (with respect to the vacuum) with the product of the PCT transformation and a rotation around the $a_{1}$-axis for $W$ given by (31). These properties have been proven by Bisognano and Wichmann [2] if local algebras are generated by Wightman fields [35], and a recent theorem by Borchers [4] shows that they hold with more generality.

As we said in the introduction, we shall deal with localized representations of $\mathscr{A}$, i.e. representations $\pi$ that have vacuum equivalent restriction to the casual complement of any space-like cone. Given a space-like cone $\mathscr{S}$ we identify the Hilbert space of $\pi$ with the vacuum Hilbert space $\mathscr{H}_{0}$ in such a way that $\left.\pi_{0}\right|_{\mathscr{A}\left(\mathscr{S}^{\prime}\right)}=\left.\pi\right|_{\mathscr{A}\left(\mathscr{S}^{\prime}\right)}$. We will call morphism localized in $\mathscr{S}$ such a localized representation on $\mathscr{H}_{0}$. A morphism $\varrho$ is transportable: for each space-like cone $\mathscr{L}_{1}$ there is a morphism localized in $\mathscr{S}_{1}$ and unitarily equivalent to $\varrho$. We observe that a transportable morphism localized in $\mathscr{S}$ maps the algebra of a wedge $\mathscr{A}(W), \mathscr{S} \mathrm{CW}$, into $\mathscr{R}(W)$ by essential duality, and it is unitarily implemented on $\mathscr{A}(W)$ by transportability, hence its restriction to $\mathscr{A}(W)$ can be extended to a normal endomorphism $\varrho_{W}$ of $\mathscr{R}(W)$. Moreover, if $\varrho$ were localized in a double cone and duality occurred [i.e. Eq. (3.2) held for double cones], then $\varrho$ would be an endomorphism of $\mathscr{A}^{2}$

Now we use the definition of conjugate endomorphism for von Neumann algebras to get a conjugation relation between morphisms of $\mathscr{A}$. We say that two morphisms $\varrho$ and $\varrho$ localized in $\mathscr{S}$ are weakly conjugate if for each wedge $W \supset \mathscr{S}, \varrho_{W}$ and $\bar{\varrho}_{W}$ are conjugate in the sense of Sect. 2.

Now let us consider the following local equivalence relation between morphisms: a morphism $\varrho^{\prime}$ is equivalent to a morphism $\varrho$ localized in $\mathscr{S}$ if we can transport it to a morphism $\varrho^{\prime \prime}$ localized in $\mathscr{S}$ such that, for each wedge $W \supset \mathscr{S}, \varrho_{W}^{\prime \prime}$ and $\varrho_{W}$ define the same element in $\operatorname{Sect}(\mathscr{R}(W))$ (see Sect. 2), i.e. there exists a unitary

${ }^{2}$ In general, $\varrho$ becomes an endomorphism of the universal $C^{*}$-algebra associated with the pre-cosheaf of the local von Neumann algebras on wedge regions, see Sect. 8 
$u \in \mathscr{R}(W)$ with

$$
\varrho_{W}^{\prime}=u \varrho_{W}^{\prime \prime}(\cdot) u^{*} .
$$

A class [@] of locally equivalent morphisms will be called sector and the symbol $\operatorname{Sect}(\mathscr{A})$ denotes here the set of all sectors of $\mathscr{A}$. We shall say that a sector is irreducible ${ }^{3}$ if, given a representative $\varrho$ localized in $\mathscr{S}$, for each wedge $W \supset \mathscr{S}, \varrho_{W}$ is irreducible:

$$
\varrho\left(\mathscr{R}(W)^{\prime}\right) \cap \mathscr{R}(W)=\mathbb{C} .
$$

Then, if an irreducible weak conjugate sector exists, it is unique, and $[\bar{\varrho}]=[\varrho]$ (cf. [27]).

We point out soon that if an irreducible DHR conjugate sector $\varrho$ exists (this is possible only if $\varrho$ has finite statistics) then $\varrho$ is also conjugate in our sense by [27].

With these premises we may now state our first result. As always we assume the vacuum sector to be Poincaré covariant.

Theorem 3.1. Let $\varrho$ be a morphism localized in the space-like cone $\mathscr{S}$ and covariant under the Euclidean group. There exists a conjugate morphism $\bar{\varrho}$ localized in $\mathscr{S}$, unique up to local equivalence.

The reason why only the Euclidean group appears in this statement will be clear later; in the next sections we discuss the Poincaré covariance.

By definition $\varrho$ is covariant under the group $G \subset P_{+}^{\uparrow}$ if there exists a (projective) continuous unitary representation $V$ of $G$ such that

$$
\varrho\left(\alpha_{g}(x)\right)=V_{g} \varrho(x) V_{g}^{*}, \quad x \in \mathscr{A}, \quad g \in G,
$$

where $\alpha_{g}(x) \equiv U_{g} x U_{g}^{*}$ and $U$ is the unitary representation of $G$ on the vacuum Hilbert space. Equivalently, $\varrho$ is $G$-covariant if

$$
\varrho_{g} \equiv \alpha_{g}^{-1} \circ \varrho \circ \alpha_{g}=U_{g}^{*} V_{g} \varrho(\cdot) V_{g}^{*} U_{g},
$$

namely if there exist unitaries

$$
z_{g} \equiv U_{g}^{*} V_{g}
$$

with

$$
\varrho_{g}=z_{g} \varrho(\cdot) z_{g}^{*} .
$$

In fact, $g \rightarrow z_{g}$ is a $\alpha$-cocycle and must be continuous. Moreover, $z_{g} \in \mathscr{R}\left(\mathscr{S}^{\prime} \cap g \mathscr{S}^{\prime}\right)^{\prime}$ and in particular, if $W \supset \mathscr{S} \cup g \mathscr{S}$, then $z_{g}$ belongs to $\mathscr{R}(W)$.

Thus $\varrho$ is covariant iff there exists a unitary map $g \rightarrow z_{g}$ (in fact, an $\alpha$-cocycle) such that

$$
\varrho_{g}(x)=z_{g} \varrho(x) z_{g}^{*}, \quad x \in \mathscr{A} .
$$

We note that since in the following $G$ will be a connected group, to prove covariance it is sufficient to check Eq. (3.4) with $g$ in a neighborhood $\mathscr{U}$ of the identity of $G$.

Lemma 3.2. Let $\mathscr{C}$ be a region in the Minkowski space and $u$ a unitary implementing $\varrho$ on $\mathscr{A}(\mathscr{C})$. Then $\alpha_{g}^{-1}\left(z_{g} u\right)$ implements $\varrho$ on $\mathscr{A}\left(g^{-1} \mathscr{C}\right)$.

3 This implies the morphism is irreducible as a representation. For the converse see Sect. 6 
Proof. We have

$$
\left.\operatorname{ad}\left(z_{g} u\right)\right|_{\mathscr{A}(\mathscr{G})}=\left.\operatorname{ad}\left(z_{g}\right) \circ \varrho\right|_{\mathscr{A}(\mathscr{E})}=\left.\varrho_{g}\right|_{\mathscr{A}(\mathscr{C})},
$$

therefore,

$$
\left.\alpha_{g}^{-1} \circ \operatorname{ad}\left(z_{g} u\right) \circ \alpha_{g}\right|_{\mathscr{A}\left(g^{-1} \mathscr{C}\right)}=\left.\varrho\right|_{\mathscr{A}\left(g^{-1 \mathscr{C}}\right)},
$$

that is

$$
\left.\operatorname{ad}\left(\alpha_{g}^{-1}\left(z_{g} u\right)\right)\right|_{\mathscr{A}\left(g^{-1} \mathscr{C}\right)}=\left.\varrho\right|_{\mathscr{A}\left(g^{-1} \mathscr{C}\right)} .
$$

Proof of Theorem 3.1. Let $W_{0}$ be a wedge that contains $\mathscr{S}$ and $j_{0}$ the antiautomorphism of $\mathscr{A}$

$$
j_{0}(x)=J_{0} x J_{0}, \quad x \in \mathscr{A},
$$

implemented by the modular conjugation $J_{0}$ of $\mathscr{R}\left(W_{0}\right) ; j_{0}$ is associated with the reflection $r_{0}$ in the Minkowski space with respect to the edge of $W_{0}$. Choose a unitary $u_{0}$ that implements $\varrho$ on $j_{0}\left(\mathscr{A}\left(\mathscr{S}^{\prime}\right)\right)=\mathscr{A}\left(r_{0} \mathscr{S}^{\prime}\right)$. Let $W \supset \mathscr{S}$ be another wedge and $j, r$ be as before; since $g=r_{0} \cdot r$ is an element of the Euclidean group (any element of the Euclidean group can be obtained as a product of reflections),

$$
u \equiv \alpha_{g}^{-1}\left(z_{g} u_{0}\right)
$$

implements $\varrho$ on $\mathscr{A}\left(g^{-1} r_{0} \mathscr{S}^{\prime}\right)=\mathscr{A}\left(r \mathscr{S}^{\prime}\right)$.

Now

$$
\begin{aligned}
\left.\bar{\varrho}_{W}\right|_{\mathscr{R}\left(W_{0} \cap W\right)} & =\left.\operatorname{ad}\left(j\left(u^{*}\right)\right)\right|_{\mathscr{R}\left(W_{0} \cap W\right)} \\
& =\operatorname{ad}\left(\left.j\left(\alpha_{g}^{-1}\left(u_{0}^{*} z_{g}^{*}\right)\right)\right|_{\mathscr{R}\left(W_{0} \cap W\right)}\right. \\
& =\left.\operatorname{ad}\left(j_{0}\left(u_{0}^{*}\right)\right) \circ \operatorname{ad}\left(j_{0}\left(z_{g}^{*}\right)\right)\right|_{\mathscr{R}\left(W_{0} \cap W\right)} \\
& =\left.\operatorname{ad}\left(j_{0}\left(u_{0}^{*}\right)\right)\right|_{\mathscr{R}\left(W_{0} \cap W\right)}=\left.\bar{\varrho}_{W_{0}}\right|_{\mathscr{R}\left(W_{0} \cap W\right)},
\end{aligned}
$$

where we have used that $j_{0}\left(z_{g}^{*}\right) \in \mathscr{R}\left(r_{0} \mathscr{S}^{\prime} \cap r \mathscr{S}^{\prime}\right)^{\prime}$ hence $\operatorname{ad}\left(j_{0}\left(z_{g}^{*}\right)\right)=$ id on $\mathscr{R}\left(W_{0} \cap W\right)$ $\left(\mathscr{R}\left(r_{0} \mathscr{S}^{\prime} \cap r \mathscr{S}^{\prime}\right)^{\prime}\right.$.

This shows that the $\bar{\varrho}_{W}$ form a consistent family of endomorphisms ( $W$ a wedge, $W \supset \mathscr{S}$ ) thus defines a morphism $\bar{\varrho}$ of $\mathscr{A}$ that, by definition, is a (weak) conjugate of $\varrho$.

\section{The Formula $\bar{\varrho}=j \circ \varrho \circ j$}

We present here an explicit formula for the conjugate sector in the same context of Sect. 3. In fact, we give a construction of a conjugate morphism that relates the description of the particle-antiparticle symmetry by the PCT antiunitary in a Wightman theory [35] with the DHR conjugation of sectors [15] in algebraic quantum field theory.

Theorem 4.1. Let $\varrho$ be a morphism localized in a space-like cone and covariant with respect to the Euclidean group. A representative of the weak conjugate sector is given by

$$
\bar{\varrho}=j \circ \varrho \circ j,
$$


where $j$ is the anti-automorphism of $B(\mathscr{H})$ implemented by the PCT antiunitary $\Theta$

$$
j(\cdot)=\Theta \cdot \Theta^{-1}
$$

associated with any given coordinate system.

Proof. Since $\varrho$ is rotation covariant, it is enough to show that

$$
\bar{\varrho}=j_{0} \circ \varrho \circ j_{0},
$$

where $j_{0}=J \cdot J$ is implemented by the modular conjugation $J$ associated to $\mathscr{R}(W)$ and $W$ is a wedge that contains the localization region $\mathscr{S}$ of $\varrho$.

To simplify the argument we shall use the time-slice axiom, that can be avoided by a more refined argument (see Sect. 8). Note that the conjugate morphism $\varrho$ constructed in the proof 'of Theorem 3.1 is localized in $\mathscr{S}$, hence, by additivity and the time-slice axiom, it is determined by

$$
\bar{\varrho}=\left\{\begin{array}{lll}
\operatorname{ad}\left(j_{0}\left(u^{*}\right)\right) & \text { on } & \mathscr{A}\left(r \mathscr{S}^{\prime}\right), \\
\mathrm{id} & \text { on } & \mathscr{A}\left(\mathscr{S}^{\prime}\right),
\end{array}\right.
$$

where $u$ is a unitary that implements $\varrho$ on $\mathscr{A}\left(r \mathscr{S}^{\prime}\right)$ and $r$ is the reflection around the edge of $W$.

On the other hand, $j_{0} \circ \varrho \circ j_{0}$ is localized in $r \mathscr{S}^{\prime}$ and is determined by

$$
j_{0} \circ \varrho \circ j_{0}=\left\{\begin{array}{lll}
\mathrm{id} & \text { on } & \mathscr{A}\left(\mathscr{S}^{\prime}\right), \\
\operatorname{ad}\left(j_{0}(u)\right) & \text { on } & \mathscr{A}\left(r \mathscr{S}^{\prime}\right) .
\end{array}\right.
$$

Therefore,

$$
\operatorname{ad}\left(j_{0}(u)\right) \circ \bar{\varrho}=j_{0} \circ \varrho \circ j_{0}
$$

that shows $j_{0} \circ \varrho \circ j_{0}$ to be equivalent to $\bar{\varrho}$.

We note that other wedges $W$ give as representatives of the conjugate sector the morphisms $j_{W} \circ \varrho \circ j_{W}$, where $j_{W}$ is the antiautomorphism of $\mathscr{A}$ associated with the modular conjugation of the algebra $\mathscr{R}(W)$. The morphisms $j_{W} \circ \varrho \circ j_{W}$ are unitarily equivalent iff the morphism $\varrho$ is Euclidean covariant.

Let $r$ be the symmetry $r(x)=-x$ of the Minkowski space, and $\vartheta$ the automorphism of the Poincare group $P_{+}^{\uparrow}$ given by

$$
\vartheta(g)=r \cdot g \cdot r, \quad g \in P_{+}^{\uparrow} .
$$

Given a unitary representation $U$ of $P_{+}^{\uparrow}$ one obtains a new representation $U^{v} \equiv U \circ \vartheta$ of $P_{+}^{\uparrow}$.

The following corollary is a stronger form of results in $[3,15]$.

Corollary 4.2. Let $\varrho$ be a Poincaré covariant morphism and $U_{\varrho}$ the corresponding unitary implementation of $P_{+}^{\uparrow}$. The conjugate morphism is also Poincaré covariant and $U_{\bar{\varrho}}$ is equivalent to the conjugate representation of $U_{\varrho}$, in fact,

$$
U_{\bar{\varrho}}(g)=\Theta U_{\varrho}^{\vartheta}(g) \Theta, \quad g \in P_{+}^{\uparrow},
$$

where $\Theta$ is the PCT antiunitary.

In particular, $U_{\varrho}$ and $U_{\bar{\varrho}}$ have the same spectrum.

$A$ similar conclusion holds if $P_{+}^{\uparrow}$ is replaced by the Euclidean or the conformal group. 
Proof. We have

$$
\operatorname{ad}\left(U_{\varrho}(g)\right) \circ \varrho=\varrho \circ \alpha_{g}
$$

hence

$$
\operatorname{ad}\left(U_{\varrho}(g)\right)^{j} \circ \varrho^{j}=\varrho^{j} \circ \alpha_{g}^{j},
$$

where $\varrho^{j}=j \circ \varrho \circ j$ and so on. Now

$$
\alpha_{g}^{j}=j \circ \alpha_{g} \circ j=\alpha_{v(g)},
$$

therefore,

$$
\operatorname{ad}\left(\Theta U_{\varrho}(\vartheta(g)) \Theta\right) \circ \varrho^{j}=\varrho^{j} \circ \alpha_{g}
$$

as desired.

\section{Regular Nets, Simple Subfactors and Covariance}

In this section we shall show that, under a certain regularity property, every DHR sector (with finite statistics, with a global conjugate) is Poincaré covariant.

We begin to explain our assumptions here. Let $\mathcal{O}_{i}(i=1, \ldots, 4)$ be the regions of the Minkowski space $\mathbb{R}^{d}$ determined by having projections in the $a_{0}-a_{1}$ plane as in the next figure

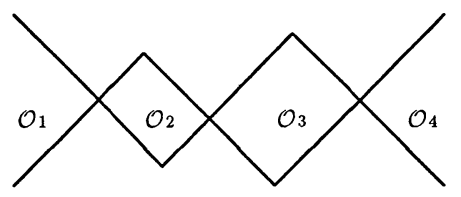

and the remaining coordinates free. In particular, $\mathcal{O}_{1}$ and $\mathcal{O}_{4}$ are wedge regions and $\mathrm{O}_{2}, \mathcal{O}_{3}$ are cylinders that projects onto double cones in the $a_{0}-a_{1}$ plane.

We shall say that the net $\mathscr{A}(\mathcal{O})$ is regular if the $C^{*}$-algebra associated with the union of the $\mathcal{O}_{i}$ is irreducible, i.e.

$$
\mathscr{A}(\mathscr{C})^{\prime \prime}=\mathscr{B}(\mathscr{H})
$$

for any region $\mathscr{C}=\bigcup \mathcal{O}_{i}$ as in the figure.

Notice that the analogous property with 3 regions would follow by Haag duality and the factoriality of the von Neumann algebra of a double cone in two space-time dimensions [a factor and its commutant generate $B(\mathscr{H})$ ].

Nets associated with free fields, hence with locally Fock fields in two dimensions, are regular (cf. e.g. the explicit calculation in [21, Appendix]) and we have no examples of a non-regular net that fulfills Haag duality.

We shortly digress now to recall the main features of simple subfactors [29]. Let $M$ be a factor; a subfactor $N$ of $M$ is simple if the identity correspondence of $M$ restricts to an irreducible $N-N$ correspondence, in other words

$$
N \vee J N J=B(\mathscr{H}),
$$

where $J$ is any modular conjugation of $M$. 
If $M^{\prime}$ is a simple subfactor of $N^{\prime}$ we say that $N$ is a cosimple subfactor of $M$. A simple subfactor $N$ of $M$ determines the automorphisms of $M$, hence if $N$ is a cosimple subfactor of $M, N$ has trivial normalizer in $M$,

$$
u \text { unitary of } M, u N u^{*}=N \Rightarrow u \in N \text {. }
$$

This is the property relevant to our situation.

Lemma 5.1. Let $\mathscr{A}(\mathcal{O})$ be a regular net and $W_{1} \subset W$ be an inclusion of wedge regions, where $W_{1}$ and $W^{\prime}$ have positive distance. Then $\mathscr{R}\left(W_{1}\right)$ is a cosimple subfactor of $\mathscr{R}(\mathcal{O})^{\prime} \cap \mathscr{R}(W)$, with $\mathcal{O} \equiv W_{1}^{\prime} \cap W$.

Proof. We have to show that $\mathscr{R}(\mathcal{O}) \vee \mathscr{R}(W)^{\prime}$ is a simple subfactor of $\mathscr{R}\left(W_{1}\right)^{\prime}$ or, by essential duality, that $\mathscr{R}(\mathcal{O}) \vee \mathscr{R}\left(W^{\prime}\right)$ is a simple subfactor of $\mathscr{R}\left(W_{1}^{\prime}\right)$.

By the Bisognano-Wichmann theorem the modular conjugation $J$ of $\mathscr{R}\left(W_{1}\right)$ is associated to a reflection of $W_{1}$ onto $W_{1}^{\prime}$ mapping $\mathcal{O}, W$ onto regions $\mathcal{O}_{0}, W_{0}$. With $N \equiv \mathscr{R}(\mathcal{O}) \vee \mathscr{R}\left(W^{\prime}\right)$ we have to show that

$$
N \vee J N J=\mathscr{R}(\mathcal{O}) \vee \mathscr{R}\left(W^{\prime}\right) \vee \mathscr{R}\left(\mathcal{O}_{0}\right) \vee \mathscr{R}\left(W_{0}^{\prime}\right)
$$

is irreducible, that is true by the regularity assumption.

In the following theorem we shall make use of the time-slice axiom.

Theorem 5.2. Let $\mathscr{A}(\mathcal{O})$ be a regular net and [@] a sector with finite statistics. We have:

a) [@] is covariant under the Poincaré group with positive energy.

b) If [@] is localized in a double cone and the vacuum sector is conformally covariant, then [@] is covariant under the proper conformal group.

Proof. To prove part a) we may assume that $\varrho$ is localized in a proper subwedge $W_{0}$ of a wedge $W$. If $\alpha_{t}, t \in \mathbb{R}$, are the automorphisms of $\mathscr{R}(W)$ implemented by the pure Lorentz transformation leaving $W$ invariant (boosts), then all the morphisms

$$
\varrho_{t} \equiv \alpha_{t} \circ \varrho \circ \alpha_{-t}, \quad|t|<1,
$$

are localized in a proper subwedge $W_{1}$ of $W$. With $\mathcal{O}=W_{1}^{\prime} \cap W$, in particular, $\varrho_{t}$ acts identically on $\mathscr{R}(\mathcal{O}),|t|<1$.

As in the proof of Theorem 7.1, finite statistics gives rise, by BisognanoWichmann theorem and Connes-Radon-Nikodym theorem, to a unitary $z_{t} \in \mathscr{R}(W)$ with

$$
\varrho_{t}(x)=z_{t} \varrho(x) z_{t}^{*}, \quad t \in \mathbb{R}, \quad x \in \mathscr{R}(W) .
$$

In particular,

$$
z_{t} \in \mathscr{R}(\mathcal{O})^{\prime} \cap \mathscr{R}(W), \quad|t|<1 .
$$

We shall show that $z_{t}$ normalizes $\mathscr{R}\left(W_{1}\right),|t|<1$, thus, by Lemma $5.1, z_{t} \in \mathscr{R}\left(W_{1}\right)$ and the time-slice axiom will entail that (5.1) holds for all $x \in \mathscr{A}$, namely $\varrho$ is $\alpha_{t}$-covariant. Since the boosts with respect to all wedge regions generate the Poincare group we conclude the proof. To show that $z_{t}$ normalizes $\mathscr{R}\left(W_{1}\right)$ note first that

$$
z_{t} \varrho\left(\mathscr{R}\left(W_{1}\right)\right) z_{t}^{*}=\varrho_{t}\left(\mathscr{R}\left(W_{1}\right)\right) \subset \mathscr{R}\left(W_{1}\right)
$$

because $\varrho_{t}$ is localized in $W_{1}$. On the other hand, $\mathscr{R}\left(W_{1}\right)$ is generated by $\varrho\left(\mathscr{R}\left(W_{1}\right)\right)$ and the Jones projection $e$ [24]; we use here that $\varrho$ has finite statistics, hence finite index [26]. But $e=v v^{*}$, where $v$ is an isometry that intertwines $\varrho \circ \varrho$ and the identity. 
Since $\varrho$ is globally defined and can be chosen localized in $W_{0}, e$ belongs to $\mathscr{R}\left(W_{0}\right)$ by essential duality. By the same reasoning $v_{t}=z_{t} v z_{t}^{*}$ intertwines $\varrho_{t} \circ \bar{\varrho}_{t}$ and the identity and the Jones projection $e_{t}=v_{t} v_{t}^{*}$ for $\varrho_{t}\left(\mathscr{R}\left(W_{1}\right)\right)$ belongs to $\mathscr{R}\left(W_{1}\right)$, $|t|<1$; since $e_{t}=z_{t} e z_{t}^{*}, z_{t}$ normalizes $\mathscr{R}\left(W_{1}\right)$.

Finally, the spectrum condition is obtained in the finite statistics case by the original argument in [15].

b) may be obtained as is in a) by using the geometrical description of the modular group of $\mathscr{R}(\mathcal{O})$ when $\mathcal{O}$ is a double cone [21]. The corresponding oneparameter transformation groups generate in fact the connected component of the identity of the conformal group, i.e. the proper conformal group.

Corollary 5.3. Let $\mathscr{A}(\mathcal{O})$ be a regular net. A sector $[\varrho]$ has finite statistics iff there exists a DHR conjugate $[\bar{\varrho}]$.

Proof. If $\varrho$ exists then $\varrho$ has finite statistics [27]. Conversely, if $\varrho$ has finite statistics, $\varrho$ is Poincaré covariant by Theorem 5.2, hence a DHR conjugate exists $[15,18]$.

\section{Split Property, Duality and Covariance}

In this section we assume the split property for space-like cones and the duality property for causally complete contractible regions. Under these general hypotheses the arguments in Sect. 3 can be reversed and the results in Sect. 5 hold in a more stringent form without the regularity condition.

More explicitly let $W$ be a wedge region and $\mathscr{S}$ a space-like cone properly contained in $W$ (namely the closure of $\mathscr{S}$ is contained in the interior of $W$ ). We shall assume the net $\mathscr{A}$ to have the split property: $\mathscr{R}(\mathscr{S}) \subset \mathscr{R}(W)$ is a split inclusion of von Neumann algebras [12] for each $\mathscr{S}$ and $W$ as above, namely there exists a type $I$ factor $F$ such that

$$
\mathscr{R}(\mathscr{S}) \subset F \subset \mathscr{R}(W) .
$$

This is an analogue of the split property for double cones (see [10,12]). It holds in the free massive field, but cannot occur in a dilation invariant theory [13].

We further assume that duality holds:

$$
\mathscr{R}(\mathscr{C})^{\prime}=\mathscr{R}\left(\mathscr{C}^{\prime}\right)
$$

for all contractible regions $\mathscr{C}$ such that $\mathscr{C}^{\prime \prime}=\mathscr{C}$ (see [1] for the proof of this property in the free field case). Indeed, we need to assume (6.2) only for regions $\mathscr{C}$ of the form $\mathscr{C}=\mathscr{S}^{\prime} \cap W$. This implies essential duality (taking $\mathscr{S}$ empty) and duality for spacelike cones (using the split property). The result of the following lemma contains the only consequence of the duality and the split property we will use in this section.

Lemma 6.1. Let $\mathscr{S}, W$ be as above. Then

$$
\mathscr{R}(\mathscr{C})^{\prime} \cap \mathscr{R}(W)=\mathscr{R}(\mathscr{S})
$$

with $\mathscr{C}=\mathscr{S}^{\prime} \cap W$.

Proof. By duality, $\mathscr{R}(\mathscr{C})^{\prime}=\mathscr{R}\left(\mathscr{S} \cup W^{\prime}\right)=\mathscr{R}(\mathscr{S}) \vee \mathscr{R}\left(W^{\prime}\right)$ and, by split property, $\mathscr{R}(\mathscr{S}) \vee \mathscr{R}\left(W^{\prime}\right)$ is naturally isomorphic to the $W^{*}$ tensor product $\mathscr{R}(\mathscr{S}) \otimes \mathscr{R}\left(W^{\prime}\right)$. Then the relative commutant of $\mathscr{R}\left(W^{\prime}\right)$ in $\mathscr{R}\left(\mathscr{C}^{\prime}\right)$ is $\mathscr{R}(\mathscr{S})$. 
Lemma 6.2. Let $\varrho$ and $\varrho^{\prime}$ be morphisms localized in a space-like cone $\mathscr{S}$ properly contained in the wedge $W$. If the endomorphisms $\varrho_{W}$ and $\varrho_{W}^{\prime}$ defined in Sect. 3 are equivalent, then $\varrho$ and $\varrho^{\prime}$ are unitarily equivalent as representations.

Proof. By the hypotheses there exists $u \in \mathscr{R}(W)$ such that ad $u \circ \varrho_{W}=\varrho_{W}^{\prime}$. By the localization of $\varrho$ and $\varrho^{\prime}$ we see that ad $u$ acts as the identity on $\mathscr{R}\left(\mathscr{S}^{\prime} \cap W\right)$, hence, using Lemma 6.1, $u \in \mathscr{R}\left(\mathscr{S}^{\prime} \cap W\right)^{\prime} \cap \mathscr{R}(W)=\mathscr{R}(\mathscr{S})$. Then ad $u \circ \varrho$ and $\varrho^{\prime}$ coincide on $\mathscr{A}\left(\mathscr{S}^{\prime}\right)$ and on $\mathscr{A}(W)$, hence on the quasilocal $C^{*}$-algebra $\mathscr{A}$.

Proposition 6.3. If $\varrho$ is a morphism of $\mathscr{A}_{0}$, then

a) @ is irreducible (Eq. (3.3)) iff it is irreducible as a representation of $\mathscr{A}$.

b) A morphism $\varrho_{1}$ is locally equivalent to $\varrho$ iff it is unitarily equivalent to $\varrho$ as a representation.

c) If $\varrho$ has finite statistics, $\varrho$ is a weak conjugate iff it is a DHR conjugate of $\varrho$.

Proof. a) Let $\varrho$ be an irreducible representation of $\mathscr{A}$, choose a wedge $W$ that properly contains the localization space-like cone $\mathscr{S}$ and take $x \in \varrho_{W}(\mathscr{R}(W))^{\prime}$ $\cap \mathscr{R}(W)$. As in Lemma $6.2 x$ belongs to $\mathscr{R}(\mathscr{S})$. Now take any wedge $W_{1} \supset W$ and consider the morphism $\varrho_{W_{1}}$. We have:

$$
\varrho_{W_{1}}\left(\mathscr{R}\left(W_{1}\right)\right)^{\prime}=\varrho_{W_{1}}\left(\mathscr{R}\left(\mathscr{S}^{\prime} \cap W_{1}\right) \vee \mathscr{R}(W)\right)^{\prime}=\mathscr{R}(\mathscr{S}) \vee \mathscr{R}\left(W_{1}\right)^{\prime} \vee \varrho_{W}(\mathscr{R}(W))^{\prime},
$$

therefore, $x \in \varrho_{W_{1}}\left(\mathscr{R}\left(W_{1}\right)\right)^{\prime}$. By the arbitrariness of $W_{1}, x \in \varrho(\mathscr{A})^{\prime}$, hence it is a multiple of the identity. The converse is obvious.

b) If $\varrho$ and $\varrho_{1}$ are locally equivalent and $\varrho$ is localized in $\mathscr{S}$, we find a morphism $\varrho^{\prime}$ unitarily equivalent to $\varrho_{1}$ and localized in $\mathscr{S}$ such that for each wedge $W$ containing $\mathscr{S} \varrho_{W}$ is equivalent to $\varrho_{W}^{\prime}$. By Lemma 6.2 ad $u \circ \varrho=\varrho^{\prime}$. The reverse implication is immediate as before.

c) If $\varrho$ is a weak conjugate of $\varrho$, both localized in $\mathscr{S}$, and $W$ is a wedge properly containing $\mathscr{S}$, we get $v \in \mathscr{R}(W)$ satisfying $\varrho \circ \varrho(x) v=v x$ for all $x$ in $\mathscr{R}(W)$. As above, $v$ turns out to be localized in $\mathscr{R}(\mathscr{S})$, hence the previous equation holds for each $x$ in $\mathscr{A}$.

Theorem 6.4. Let $\varrho$ be an irreducible morphism localized in a space-like cone $\mathscr{S}$. If the split and duality properties (6.1) and (6.2) hold, the following are equivalent:

(i) $\varrho$ is covariant under the Euclidean group.

(ii) $\varrho$ has a weak conjugate $\varrho$ localized in $\mathscr{S}$.

Moreover, if @ has finite statistics, @ is Poincaré covariant.

Proof. (i) $\Rightarrow$ (ii) is shown in Theorem 3.1.

(ii) $\Rightarrow$ (i). If $\varrho$ is a weak conjugate of $\varrho$ and $W$ a wedge that properly contains $\mathscr{S}$, we take $U$ implementing $\varrho$ on $J_{W} \mathscr{R}\left(\mathscr{S}^{\prime}\right) J_{W}$ and consider the morphism $\bar{\varrho}^{\prime} \equiv \operatorname{ad}\left(J_{W} U^{*} J_{W}\right) \circ j_{W} \circ \varrho \circ j_{W}$. Since $\bar{\varrho}_{W}^{\prime}$ is equivalent to $\bar{\varrho}_{W}$ and $\bar{\varrho}^{\prime}$ is unitarily equivalent to $j_{W} \circ \varrho \circ j_{W}$, by Lemma $6.2 \varrho$ is unitarily equivalent to $j_{W} \circ \varrho \circ j_{W}$. This gives us the equivalence of all the morphisms $j_{W} \circ \varrho \circ j_{W}$ if $W \supset \mathscr{S}$, hence the equivalence of $\varrho$ and $j_{W_{1}} \circ j_{W_{2}} \circ \varrho \circ j_{W_{2}} \circ j_{W_{1}}$ when $W_{1}$ and $W_{2}$ properly contain $\mathscr{S}$. Since any $\alpha_{g}$ with $g$ in a suitable neighbourhood of the identity of the Euclidean group can be written as $j_{W_{1}} \circ j_{W_{2}}$ with $W_{1}$ and $W_{2}$ properly containing $\mathscr{S}$, we get the covariance of $\varrho$.

If $\varrho$ has finite statistics it is easy to see that the proof of Theorem 5.2a) works substituting regularity with the result of Lemma 6.1 . 


\section{Theories with Countably Many Sectors}

In this section we prove the covariance of the superselection sectors with finite statistics in the case when the family of sectors is countable. We use without further mention the general assumptions stated in Sect. 3, but neither the regularity condition nor assumptions in Sect. 6 are needed here.

Theorem 7.1. If $\operatorname{Sect}(\mathscr{A})$ is countable, then for any $[\varrho] \in \operatorname{Sect}(\mathscr{A})$ with finite statistics, the morphism @ is Poincaré covariant with spectrum condition.

Proof. We may assume that $\varrho$ is localized in the wedge region $W$. Let $\alpha_{t}, t \in \mathbb{R}$, be the one parameter group of automorphisms of $\mathscr{A}$ implemented by $V(2 \pi t)$, where $V$ is the unitary representation of the boosts leaving $W$ invariant. The restriction of $\alpha$ to $\mathscr{A}(W)$ extends to the modular group $\sigma^{W_{0}}$ of the von Neumann algebra $\mathscr{M} \equiv \mathscr{R}(W)[2]$.

Since we have

$$
\operatorname{Ind}(\varrho(\mathscr{M}), \mathscr{M})=d(\varrho)^{2}<\infty
$$

there exists a normal faithful conditional expectation $\varepsilon$ of $\mathscr{M}$ onto $\varrho(\mathscr{M})$. Let $\varphi$ be a normal faithful state of $\mathscr{M}$ leaving $\varepsilon$ invariant, namely $\varphi=\varphi_{0} \cdot \varepsilon$ for some faithful $\varphi_{0} \in \varrho(\mathscr{M})_{*}^{+}$. Then $\sigma^{\varphi}$ leaves $\varrho(\mathscr{M})$ globally invariant and restricts to $\sigma^{\varphi_{0}}$ on $\varrho(\mathscr{M})$. We have

$$
\sigma^{\varphi \cdot \varrho}=\varrho^{-1} \circ \sigma^{\varphi_{0}} \circ \varrho,
$$

namely

$$
\varrho \circ \sigma^{\varphi \cdot \varrho}=\sigma^{\varphi} \circ \varrho .
$$

Let

$$
u_{t}=\left(D \varphi: D \omega_{0}\right)_{t}, \quad v_{t}=\left(D \varphi \cdot \varrho: D \omega_{0}\right)_{t} \in \mathscr{M}
$$

be the Connes-Radon-Nikodym cocycles [11], then

$$
\sigma_{t}^{\varphi}=u_{t} \sigma_{t}^{\omega_{0}}(\cdot) u_{t}^{*}, \quad \sigma_{t}^{\varphi \cdot \varrho}=v_{t} \sigma_{t}^{\omega_{0}}(\cdot) v_{t}^{*}
$$

so that

$$
\varrho\left(v_{t} \sigma_{t}^{\omega_{0}}(\cdot) v_{t}^{*}\right)=u_{t} \sigma_{t}^{\omega_{0}}(\varrho(\cdot)) u_{t}^{*}
$$

namely

$$
z_{t} \varrho(x) z_{t}^{*}=\alpha_{t} \circ \varrho \circ \alpha_{-t}(x)
$$

for all $x \in \mathscr{M}$ with $z_{t}=u_{t}^{*} \varrho\left(v_{t}\right) \in \mathscr{M}$.

We have to show that

$$
\alpha_{t} \circ \varrho \circ \alpha_{-t}=z_{t} \varrho(\cdot) z_{-t} \text { on } \mathscr{A} ;
$$

to this end note that $\varrho_{t} \equiv \alpha_{t} \circ \varrho \circ \alpha_{-t}$ is a representative of a sector for every $t \in \mathbb{R}$ and the group

$$
\mathscr{G}=\left\{t \in \mathbb{R} \mid\left[\varrho_{t}\right]=[\varrho]\right\}
$$

is dense in $\mathbb{R}$ since otherwise $\left\{\left[\varrho_{t}\right], t \in \mathbb{R}\right\}$ would be uncountable. 
Let $w_{t} \in B\left(\mathscr{H}_{0}\right)$ the unitary such that

$$
\varrho_{t}=w_{t} \varrho(\cdot) w_{t}^{*}, \quad t \in \mathscr{G} .
$$

Since $\varrho$ is localized in a proper subwedge of $W$, there exists a proper subwedge $W_{0}$ of $W$ such that all $\varrho_{t}$ are localized in $W_{0}$ for $|t|<1$. It follows by essential duality that

$$
w_{t} \in \mathscr{R}\left(W_{0}\right), \quad|t|<1 \text {. }
$$

By (7.1) and (7.2) we have that

$$
w_{t}=c_{t} z_{t}, \quad t \in \mathscr{G},
$$

with $c_{t} \in \varrho(\mathscr{M})^{\prime} \cap \mathscr{M}$ that is a finite dimensional algebra. Given any $t \in(-1,1)$ let $t_{n} \in \mathscr{G}, t_{n} \rightarrow t$; since $\varrho(\mathscr{M})^{\prime} \cap \mathscr{M}$ is finite dimensional we may assume that $c_{t_{n}}$ is norm convergent; since $z_{t_{n}} \rightarrow z_{t}$ strongly, $w_{t}$ is strongly convergent to a unitary $w_{t_{0}}$. It follows that

$$
\begin{aligned}
\varrho_{t_{0}}(x) & =\lim _{t \rightarrow t_{0}} \varrho_{t}(x)=\lim _{t \rightarrow t_{0}} w_{t} \varrho(x) w_{t}^{*} \\
& =w_{t_{0}} x w_{t_{0}}^{*}, \quad x \in \mathscr{A} .
\end{aligned}
$$

Namely $t_{0} \in \mathscr{G}$ and $\mathscr{G}=\mathbb{R}$. By composing different boosts we may generate all the Poincaré group, hence $\varrho$ is Poincaré covariant.

Corollary 7.2. In Theorem 7.1 assume further that the vacuum sector is conformally covariant. Then $\varrho$ is covariant with respect to the proper conformal group.

Proof. Immediate by the argument in the proof of Theorem 5.2.

\section{Covariance and Conjugates for Conformal Theories on the Circle}

As in $[6,17]$ we consider the local algebras on $S^{1}$ associated with chiral fields of two-dimensional conformal theories. Let $\mathscr{A}: I \rightarrow \mathscr{A}(I)$ be a map from proper intervals $I$ (i.e. $I$ and its complement have non-empty interior) of $S^{1}$ to von Neumann algebras $\mathscr{A}(I)$ on a Hilbert space $\mathscr{H}_{0}$, together with a unitary, positive energy ${ }^{4}$ representation of the Möbius group $M_{0}$ on $\mathscr{H}_{0}$ leaving the vacuum vector invariant. They should satisfy the following properties:

$$
\begin{gathered}
I \subset J \Rightarrow \mathscr{A}(I) \subset \mathscr{A}(J) \quad \text { (isotony), } \\
I \cap J=\phi \Rightarrow \mathscr{A}(I) \subset \mathscr{A}(J)^{\prime} \quad \text { (locality), } \\
U(g) \mathscr{A}(I) U(g)^{*}=\mathscr{A}(g I), \quad g \in M_{0} \quad \text { (covariance). }
\end{gathered}
$$

We will assume local algebras to be generated by Wightman fields with Bisognano-Wichmann hypotheses [2]. Hence, if $I$ is a proper interval, the modular automorphism group of $\mathscr{A}(I)$ with respect to the vacuum corresponds to a one parameter subgroup of $M_{0}$, the modular conjugation corresponds to a reflection

4 The energy operator is defined here as the generator of the rotation subgroup 
and, by the Tomita commutation theorem, Haag duality holds:

$$
\mathscr{A}\left(I^{\prime}\right)=\mathscr{A}\left(I^{\prime}{ }^{5},\right.
$$

where $I^{\prime}$ denotes the interior of the complement of $I$.

A positive energy covariant representation of $\mathscr{A}$ is a family of representations $\pi^{I}$ of the algebras $\mathscr{A}(I)$ on a Hilbert space $\mathscr{H}_{\pi}$, and a unitary positive energy representation $U^{\pi}$ of the covering group $M$ of $M_{0}$ on $\mathscr{H}_{\pi}$, with the following properties:

$$
\begin{array}{cl}
\left.J \subset I \Rightarrow \pi^{I}\right|_{\mathscr{Q}(J)}=\pi^{J} & \text { (isotony), } \\
\operatorname{ad} U_{g}^{\pi} \circ \pi^{J}=\pi^{\dot{g} I} \circ \operatorname{ad} U_{\dot{g}} & \text { (covariance), }
\end{array}
$$

where $g \in M \rightarrow \dot{g} \in M_{0}$ is the covering homomorphism. Covariance and positive energy imply [6] that $\pi^{I}$ is unitarily equivalent to the identity (vacuum representation $\pi_{0}$ ) if $I$ is a proper interval, hence we choose a proper interval $I_{0}$ and identify $\mathscr{H}_{0}$ and $\mathscr{H}_{\pi}$ is such a way that $\pi^{I^{\prime}}=\left.\pi_{0}\right|_{\mathscr{A}\left(I_{0}^{\prime}\right)}$. We will say that $\pi$ is localized in $I_{0}$. A local equivalence class of representations of $\mathscr{A}$ obeying (8.2) is a sector (see Sect. 3).

To describe in a more conventional way the superselection structure, Fredenhagen [17] has defined a global $C^{*}$-algebra that plays the same role as the quasilocal $C^{*}$-algebra on the Minkowski space. Since proper intervals of $S^{1}$ do not form a direct set, $\mathscr{A}$ could not be defined as an inductive limit. We digress here to give an equivalent definition of such an algebra introducing the notion of pre-cosheaf [5]. A pre-cosheaf $\mathscr{B}$ of $C^{*}$-algebras on $S^{1}$ is a covariant functor between the category of open subsets of $S^{1}$ with inclusions and the category of $C^{*}$-algebras. Namely $\mathscr{B}$ is a map

$$
I \rightarrow \mathscr{B}(I)
$$

associating a $C^{*}$-algebra with each open subset of $S^{1}$ and homomorphisms

$$
\varepsilon_{I, J}^{\mathscr{B}}: \mathscr{B}(I) \rightarrow \mathscr{B}(J) \text {, where } I \subset J .
$$

If $\mathscr{B}$ were defined only on intervals (or proper intervals) we would say that $\mathscr{B}$ is a pre-cosheaf on intervals (or proper intervals). A cosheaf map $\phi$ between the precosheaves $\mathscr{B}$ and $\mathscr{C}$ is a family of morphisms $\phi_{I}, I$ open subset of $S^{1}$, such that if $I \subset J$

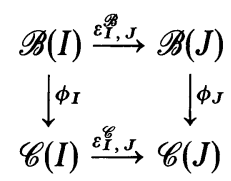

is a commuting diagram.

\footnotetext{
5 As a consequence of the Bisognano-Wichmann theorem, in a conformal theory of any spacetime dimension $d$, the modular conjugation of $\mathscr{R}(\mathcal{O})$ is associated with the ray inversion map, where $\mathcal{O}$ is a (unital) double cone [21]. As pointed out recently in [9], formula (8.1) does not hold in general on the cut circle $S^{1} \backslash\{p t\}=\mathbb{R}$. This phenomenon is analogous to the case of the free massless field, where time-like duality (if $d$ is even and twisted time-like duality if $d$ is odd) holds: $\mathscr{A}(\mathcal{O})^{\prime}=\mathscr{A}\left(\mathcal{O}^{t}\right)$, with $\mathcal{O}^{t}$ the time-like complement of $\mathcal{O}$, but $\mathscr{A}\left(\mathcal{O}^{t}\right)$ is not defined by additivity $(d>2)$ but by fields smeared on testfunctions not vanishing at infinity [21]
} 
Now the map $I \rightarrow \mathscr{A}(I)$ given by the local observable algebras is a pre-cosheaf on the proper open intervals of $S^{1}$ if the $\varepsilon_{I, J}^{\mathscr{A}}$ are the inclusions. We look for an extension of $\mathscr{A}$ to a pre-cosheaf $\tilde{A}$ on $S^{1}$, that is universal. Namely, if $\mathscr{B}$ is any other extension of $\mathscr{A}$ on $S^{1}$ there exists a unique cosheaf map $\eta: \tilde{A} \rightarrow \mathscr{B}$ on the intervals [i.e. (8.3) holds if $I$ and $J$ are intervals], such that

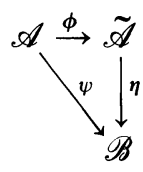

is a commutative diagram, where $\phi$ and $\psi$ are the cosheaf embeddings of $\mathscr{A}$ into $\tilde{\mathscr{A}}$ and $\mathscr{B}$. The universal $C^{*}$-algebra $\mathscr{A}_{0}=\tilde{\mathscr{A}}\left(S^{1}\right)$ is unique up to isomorphisms. To construct $\tilde{\mathscr{A}}$ let $\Psi$ be the direct sum of all cosheaf maps $\psi$ on proper intervals from $\mathscr{A}$ to pre-cosheaves $\mathscr{B}$ on $S^{1}$ extending $\mathscr{A}$ as above. Then $\Psi$ maps $\mathscr{A}(I)$ into the direct sum of all the algebras $\mathscr{B}(I)$. The $C^{*}$-algebra $\mathscr{A}_{0}$ is the $C^{*}$-subalgebra generated by $\varepsilon_{I, S^{1}}(\Psi(\mathscr{A}(I))$ as $I$ varies in the proper intervals. Since the vacuum representation provides an extension to a pre-sheaf on $S^{1}$ by additivity, the $\mathscr{A}(I)$ are faithfully embedded in $\mathscr{A}_{0}$ and we identify the local subalgebras with their image in $\mathscr{A}_{0}$.

It follows from the definition that representations of the $\mathscr{A}(I)$ 's extend uniquely to representations of $\mathscr{A}_{0}$, that is the universality property of Fredenhagen. The cosheaf maps ad $U_{g}$ give therefore rise to an action $g \rightarrow \alpha_{g}$ of $M_{0}$ on the universal algebra $\mathscr{A}_{-0}$.

We denote by $\dot{\alpha}$ the action of $M$ on $\mathscr{A}$ defined by $\dot{\alpha}_{g}=\alpha_{g}$. In the following our analysis slightly differs from that in [17] in the way be build up an endomorphism of $\mathscr{A}$ describing a representation $\pi$. We need a canonical construction that we obtain by the covariance of $\pi$. We shall use the correspondence between localized endomorphisms and localized cocycles (Roberts [34]) and describe endomorphisms and their conjugates in terms of cocycles (in particular, in cohomology). The map

$$
g \in M \rightarrow V_{g}=U_{\dot{g}} U_{g^{-1}}^{\pi}
$$

is a cocycle with respect to the action $g \in M \rightarrow \operatorname{ad} U_{\dot{g}}$, that is

$$
V_{g \cdot h}=\operatorname{ad} U_{\dot{g}}\left(V_{h}\right) V_{g} .
$$

By the covariance and the localization of $\pi$ we get

$$
\pi^{g I_{0}^{\prime}}=\left.\operatorname{ad} U_{g}^{\pi} \circ \pi^{I^{\prime} \circ} \circ \operatorname{ad} U_{\dot{g}}^{*}\right|_{\mathscr{A}\left(g I_{0}^{\prime}\right)}=\left.\operatorname{ad} V_{g}^{*}\right|_{\mathscr{A}\left(\dot{g} I_{0}^{\prime}\right)} .
$$

Again by the localization of $\pi$, the preceding equation implies

$$
V_{g} \in \mathscr{A}\left(I_{0} \cup \dot{g} I_{0}\right) \quad \text { if } \quad I_{0} \cup \dot{g} I_{0} \quad \text { is a proper interval. }
$$

We will denote by $\mathscr{U}$ the open set in $M$ defined by

$$
\mathscr{U}=\left\{g \in M / I_{0} \cup \dot{g} I_{0} \text { is a proper interval }\right\} .
$$

Now we will define a $\dot{\alpha}$-cocycle in $\mathscr{A}$. If $g \in \mathscr{U}$, since $\pi_{0}$ is locally faithful, there exists a unique $w_{g} \in \mathscr{A}\left(I_{0} \cup \dot{g} I_{0}\right)$ such that

$$
\pi_{0}\left(w_{g}\right)=V_{g} .
$$


In particular, if $g, h, g \cdot h \in \mathscr{U}$, Eq. (8.4) becomes

$$
w_{g \cdot h}=\dot{\alpha}_{g}\left(w_{h}\right) w_{g} .
$$

Proposition 8.2. The map $g \in \mathscr{U} \rightarrow w_{g}$ extends to a unitary $\dot{\alpha}$-cocycle on $M$ with values in $\mathscr{A}_{0}$ and

$$
\pi_{0}\left(w_{g}\right)=V_{g}, \quad \forall g \in M .
$$

Proof. The group generated by $\mathscr{U}$ is open and closed, hence, by connectedness, coincides with $M$. Therefore, each $g \in M$ can be written as

$$
g=\prod_{i=1}^{n} g_{i}, \quad g_{i} \in \mathscr{U} .
$$

If we define in $\mathscr{A}_{0}$ the unitary $w_{g}=w_{g_{1}} \cdot \alpha_{g_{1}}\left(w_{g_{2}}\right) \ldots \alpha_{g_{1} \ldots g_{n-1}}\left(w_{g_{n}}\right)$, a standard deformation argument shows that the definition does not depend on the choice of the $g_{n}$ 's, by the simple connectedness. The cocycle property follows from the definition of $w$.

Notice that $w_{g}$ is an $\dot{\alpha}$-cocycle localized in $I_{0}$, i.e.

$$
\begin{gathered}
w_{g \cdot h}=\dot{\alpha}_{g}\left(w_{h}\right) w_{g}, \quad g, h \in M, \\
w_{g} \in \mathscr{A}^{\mathrm{d}}\left(I_{0} \cup \dot{g} I_{0}\right),
\end{gathered}
$$

where $\mathscr{A}^{\mathrm{d}}(I) \equiv \mathscr{A}\left(I^{\prime}\right)^{\prime}$ and $I_{0} \cup \dot{g} I_{0}$ is contained in a proper interval. To assign a localized $\dot{\alpha}$-cocycle is equivalent to assign an endomorphism of $\mathscr{A}_{0}$ localized in $I_{0}$, covariant with respect to the action $\dot{\alpha}$ (cf. [34]). Given $w$ as in (8.6), the corresponding endomorphism $\varrho$ is determined by

$$
\left.\varrho\right|_{\mathscr{A}\left(g I_{0}^{\prime}\right)}=\left.\mathrm{ad} w_{g}^{*}\right|_{\mathscr{A}\left(\dot{g} I_{0}\right)} \text {. }
$$

Since any proper interval of $S^{1}$ can be written as $\dot{g} I_{0}^{\prime}$ for a suitable $g \in M$ and the homomorphisms $\left.\varrho\right|_{\mathscr{A}(I)}$ form a consistent family (coincide on intersections), the endomorphisms $\varrho$ is well defined on $\mathscr{A}_{0}$ by the universal property. By definition, we get

$$
\left.\pi\right|_{\mathscr{A}\left(g I_{0}^{\prime}\right)}=\left.\operatorname{ad} V_{g} \circ \pi_{0}\right|_{\mathscr{A}\left(g I_{0}\right)}=\left.\pi_{0} \circ \operatorname{ad} w_{g}\right|_{\mathscr{A}\left(g I^{\prime}\right)}=\left.\pi_{0} \circ \varrho\right|_{\mathscr{A}\left(g I_{0}^{\prime}\right)}, \quad \forall g \in M,
$$

hence

$$
\pi=\pi_{0} \circ \varrho \text { on } \tilde{\mathscr{A}},
$$

namely the representation $\pi$ is re-obtained by the endomorphism $\varrho$. The covariance of $\pi$ is thus encoded in the cocycle properties of $w_{g}$, and can be written

$$
\operatorname{ad} w_{g} \circ \varrho=\dot{\alpha}_{g} \circ \varrho \circ \dot{\alpha}_{g^{-1}}, \quad g \in M .
$$

Now we are able to state the analogue of Theorems 3.1 and 3.3.

Theorem 8.3. To each covariant endomorphism @ localized in a proper interval $I_{0}$, there corresponds a weak conjugate endomorphism $\varrho$ localized in $I_{0}$, namely $\left.\varrho\right|_{\mathscr{Q}(I)}$ and $\left.\varrho\right|_{\mathscr{A}(I)}$ are conjugate endomorphisms for each proper interval $I \supset I_{0}$. 
The weak conjugate sector $[\bar{\varrho}]$ is unique up to local equivalence.

An explicit formula for a representative $\bar{\varrho}^{I}$ of $[\bar{\varrho}]$ is

$$
\varrho^{I}=j_{I} \circ \varrho \circ j_{I},
$$

where $I$ is any proper interval of $S^{1}$ and $j_{I}$ is the associated reflection antiautomorphism.

To prove the theorem we will construct a family of endomorphisms in terms of a family of $\dot{\alpha}$-cocycles. Then we will show that they belong to the same sector and are conjugate to $\varrho$ in the appropriate sense. We will call reflection a diffeomorphism $r$ of $S^{1}$ that is Möbius conjugate to the diffeomorphism $z \rightarrow z^{-1}$. Notice that $r^{2}=\mathrm{id}$ and $r \notin M_{0}$. It is easy to see that each reflection leaves two points invariant, and it is determined by them. The conjugation by a reflection,

$$
g \in M_{0} \rightarrow r \cdot g \cdot r \in M_{0}
$$

is an automorphism of $M_{0}$, and extends uniquely to an automorphism of the covering group $M$. We will still use the notation $r \cdot g \cdot r$, if $g \in M$, to denote the action of this automorphism. By the Bisognano-Wichmann theorem and [21] the reflection $r_{I}$ associated to an interval $I$, i.e. the reflection such that $r I=I^{\prime}$, acts on $\mathscr{A}_{0}$ by the antiautomorphism $\dot{j}_{r}=j_{\mathrm{I}}$ implemented by the modular conjugation of the algebra $\mathscr{A}(I)$ in the vacuum representation. In particular,

$$
\begin{array}{cc}
j_{r}(\mathscr{A}(I))=\mathscr{A}(r I), & j_{I}(\mathscr{A}(I))=\mathscr{A}\left(I^{\prime}\right), \\
j_{r_{1}} \circ j_{r_{2}}=\dot{\alpha}_{r_{1} \cdot r_{2}}, & j_{r} \circ \dot{\alpha}_{g} \circ j_{r}=\dot{\alpha}_{r \cdot g} \cdot r .
\end{array}
$$

Proof. We will prove Theorem 8.3 in three steps.

a) If $h \in M$ and $r$ is a reflection, the map

$$
g \rightarrow \bar{w}_{g}^{h, r}=\dot{\alpha}_{h} \circ j_{r}\left(w_{r} \cdot h^{-1} \cdot g \cdot h \cdot r\right)
$$

is a cocycle localized in $h \cdot r I_{0}$ which corresponds to the endomorphism $\bar{\varrho}^{h, r}=\dot{\alpha}_{h} \circ j_{r} \circ \varrho \circ j_{r} \circ \dot{\alpha}_{h^{-1}}$.

In fact, we only need to prove that Eq. (8.5) holds:

$$
\begin{aligned}
& \dot{\alpha}_{g} \circ \underline{\varrho}^{h, r} \circ \dot{\alpha}_{g^{-1}}=\dot{\alpha}_{h} \circ j_{r} \circ \dot{\alpha}_{r \cdot h^{-1} \cdot g \cdot h \cdot r} \circ \varrho \circ \dot{\alpha}_{r \cdot h^{-1} \cdot g^{-1} \cdot h \cdot r} \circ j_{r} \circ \dot{\alpha}_{h^{-1}} \\
& =\dot{\alpha}_{h} \circ j_{r} \circ \operatorname{ad~} w_{r \cdot h}-1 \cdot g \cdot h \cdot r \circ \varrho \circ j_{r} \circ \dot{\alpha}_{h-1} \\
& =\operatorname{ad} \bar{w}_{g}^{h, r} \circ \bar{\varrho}^{h, r} \text {. }
\end{aligned}
$$

b) All endomorphisms $\varrho^{-h, r}$ are in the same sector:

If $g, h \in M$ and $r, s$ are reflections,

$$
\begin{aligned}
\bar{\varrho}^{h, r} & =\dot{\alpha}_{h} \circ j_{r} \circ j_{s} \circ \dot{\alpha}_{g^{-1}} \circ \bar{\varrho}^{g, s} \circ \dot{\alpha}_{g} \circ j_{s} \circ j_{r} \circ \dot{\alpha}_{h^{-1}} \\
& =\dot{\alpha}_{h \cdot r \cdot s \cdot g^{-1}} \circ \varrho^{g, s} \circ \dot{\alpha}_{g \cdot s \cdot r \cdot h}=\operatorname{ad} \bar{w}_{h \cdot r \cdot s \cdot g^{-1}}^{g, s} \circ \bar{\varrho}^{g, s} .
\end{aligned}
$$

c) $\varrho^{h, r}$ is conjugate to $\varrho$ for each $h \in M$ and for each reflection $r$ :

By a) and b) and using Lemma 2.1, it is sufficient to show that for each region $I \supset I_{0}$ there exists a unitary $u$ implementing $\varrho$ on $I$ and a pair $(h, r)$ such that

$$
\left.\operatorname{ad}\left(j_{I}\left(u^{*}\right)\right)\right|_{\mathscr{A}(I)}=\left.\bar{\varrho}^{h, r}\right|_{\mathscr{A}(I)} \text {. }
$$


Let $r$ be the reflection associated with $I$ and then choose an $h \in M$ with $h \cdot r I_{0}=I_{0}$. Hence

$$
\left.\operatorname{ad} w_{r \cdot h \cdot r}^{*}\right|_{\mathscr{A}\left(r \cdot h \cdot r I^{\prime}\right)}=\left.\varrho\right|_{\mathscr{A}\left(r \cdot h \cdot r I^{\prime}\right)}=\left.\varrho\right|_{\mathscr{A}\left(r I^{\prime}\right)},
$$

and since $r I_{0}^{\prime} \supset I$, we can choose $u=w_{r \cdot h \cdot r}^{*}$. Moreover, using the identities (7.5) and (8.7),

$$
\begin{aligned}
j_{I}\left(u^{*}\right) & =j_{r}\left(w_{r \cdot h \cdot r}\right)=j_{r} \circ \dot{\alpha}_{r \cdot h \cdot r}\left(w_{r \cdot h-1 \cdot r}^{*}\right)=\dot{\alpha}_{h} \circ j_{r}\left(w_{r \cdot h}^{*-1 \cdot r}\right) \\
& =\left(\bar{w}_{h-1}^{h, r}\right)^{*},
\end{aligned}
$$

therefore,

$$
\left.\operatorname{ad} j_{I}\left(u^{*}\right)\right|_{\mathscr{A}\left(r I_{0}^{\prime}\right)}=\left.\operatorname{ad}\left(\bar{w}_{h^{-}}^{h, r}\right)^{*}\right|_{\mathscr{A}\left(h^{-1} I_{0}^{\prime}\right)}=\left.\bar{\varrho}^{h, r}\right|_{\mathscr{A}\left(r I_{0}^{\prime}\right)} .
$$

Again since $r I_{0}^{\prime} \supset I$, we get

$$
\left.\operatorname{ad} j_{I}\left(u^{*}\right)\right|_{\mathscr{A}(I)}=\left.\bar{\varrho}^{h, r}\right|_{\mathscr{A}(I)} .
$$

Reversing the above arguments, the results of Sects. 5 and 7 extend, mutatis mutandis, to obtain the automatic Möbius covariance of sectors in the above setting. Because the treatment is analogous, we omit the details.

The analysis in this section suggests to consider a cosheaf structure [5] associated with the local algebras. This is possible, but goes beyond the purpose of this paper. Instead, we shall see in the next section the role played by the sheaf structure.

\section{Covariance and Sheaves of von Neumann Algebras}

In this section we give a first insight on a geometrical interpretation of the covariance of superselection sectors. We shall see that local algebras have a natural structure of sheaf of von Neumann algebras and covariance is equivalent to the fact that superselection sectors are sheaf maps, modulo suitable inner automorphisms.

Roberts [33] has long considered a sheaf structure associated with the local von Neumann algebras. Although his structure is closely related to ours it differs both because his von Neumann algebras (or the commutants) in general do not coincide with ours, and, more significantly, restriction maps are different.

As a consequence we obtain an abstract covariance result that contains for example the covariance with respect to further geometrical transformations in conformal theories.

Let now $X$ be a topological space. A presheaf of von Neumann algebras on $X$ is a map $\mathcal{O} \subset X \rightarrow \mathscr{A}(\mathcal{O})$ from open subsets $\mathcal{O}$ of $X$ to von Neumann algebras $\mathscr{A}(\mathcal{O})$ together with a homomorphism $\gamma_{\tilde{\theta}, \mathcal{O}}$ of $\mathscr{A}(\widetilde{\mathcal{O}})$ into $\mathscr{A}(\mathcal{O})$ for any inclusion of open sets $\mathcal{O} \subset \tilde{\mathcal{O}}$ that satisfy:

$$
\begin{gathered}
\gamma_{\mathcal{O}_{2}, \mathcal{O}_{1}} \gamma_{\mathcal{O}_{3}, \mathcal{O}_{2}}=\gamma_{\mathcal{O}_{3}, \mathcal{O}_{1}} \text { if } \mathcal{O}_{1} \subset \mathcal{O}_{2} \subset \mathcal{O}_{3}, \\
\gamma_{\mathcal{O}, \mathcal{O}}=\text { id. }
\end{gathered}
$$

The maps $\gamma_{\tilde{\mathcal{O}}, \mathcal{O}}$ are called restrictions and if $x \in \mathscr{A}(\widetilde{\mathcal{O}})$ we may also write

$$
\left.x\right|_{\mathcal{O}} \equiv \gamma_{\tilde{0}, \mathcal{O}}(x) \text {. }
$$


The presheaf $\mathscr{A}$ is a sheaf if given an open covering $\mathcal{O}=\cup \mathcal{O}_{i}$ and elements $x_{i} \in \mathscr{A}\left(\mathcal{O}_{i}\right)$ with

$$
\left.x_{i}\right|_{\mathcal{O}_{i} \cap \mathcal{O}_{j}}=\left.x_{j}\right|_{\mathcal{O}_{i} \cap \mathcal{O}_{j}}
$$

there exists a unique $x \in \mathscr{A}(\mathcal{O})$ with

$$
\left.x\right|_{\mathcal{O}_{i}}=x_{i}
$$

We shall consider the case where $X$ is the Minkowski space. For any proper open set $\mathcal{O}$, namely an open non-empty set with non-empty space-like complement $\mathcal{O}^{\prime}$, the vacuum vector is cyclic and separating for $\mathscr{A}(\mathcal{O})$ by the Reeh-Schlieder theorem, and the associated canonical endomorphism

$$
\gamma_{\tilde{\mathcal{O}}, \mathcal{O}}: \mathscr{A}(\widetilde{\mathcal{O}}) \rightarrow \mathscr{A}(\mathcal{O})
$$

provides a natural restriction map ${ }^{6}$ for any inclusion of proper sets $\mathcal{O} \subset \tilde{\mathcal{O}}$.

We shall speak of sheaves on a family of open sets if the above properties are satisfied for open sets in this family.

Next proposition shows that $\mathscr{A}$ gives rise to a sheaf on proper open sets.

Proposition 9.1. Let $\mathscr{A}$ be an additive net of von Neumann algebras on the Minkowski space. If the restriction maps

$$
\gamma_{\tilde{\mathcal{O}}, \mathcal{O}}: \mathscr{A}(\tilde{\mathcal{O}}) \rightarrow \mathscr{A}(\mathcal{O})
$$

are the canonical endomorphisms of $\mathscr{A}(\widetilde{\mathcal{O}}) \rightarrow \mathscr{A}(\mathcal{O})$ with respect to the vacuum, then $\mathscr{A}$ is a sheaf of von Neumann algebras on proper open sets.

Proof. The chain rule follows from the canonicity of the endomorphisms. To check the sheaf property let $\mathcal{O}=\bigcup_{i} \mathcal{O}_{i}$, where the $\mathcal{O}_{i}$ are proper open sets and $x_{i} \in \mathscr{A}\left(\mathcal{O}_{i}\right)$ with

$$
\left.x_{i}\right|_{\mathcal{O}_{i} \cap \mathcal{O}_{j}}=\left.x_{j}\right|_{\boldsymbol{O}_{i} \cap \Theta_{j}} .
$$

With $J_{\mathscr{O}}$ the modular conjugation of $\mathscr{A}(\mathcal{O})$ with respect to the vacuum, let

$$
y_{i}=J_{\mathscr{O}} J_{\mathfrak{O}_{i}} x_{i} J_{\mathfrak{O}_{i}} J_{\mathscr{O}} \in B(\mathscr{H}),
$$

then

$$
J_{\mathscr{O}} y_{i} J_{\mathscr{O}}=J_{\mathcal{O}_{i}} x_{i} J_{\mathcal{O}_{i}}
$$

By assumptions we have

$$
\begin{aligned}
J_{\mathcal{O}_{i} \cap \mathcal{O}_{j}} J_{\mathcal{O}_{i}} x_{i} J_{\mathscr{O}_{i}} J_{\mathcal{O}_{i} \cap \mathcal{O}_{j}} & =\left.x_{i}\right|_{\mathcal{O}_{i} \cap \mathcal{O}_{j}} \\
& =\left.x_{j}\right|_{\mathcal{O}_{i} \cap \mathcal{O}_{j}}=J_{\mathfrak{O}_{i} \cap \mathcal{O}_{j}} J_{\mathcal{O}_{j}} x_{j} J_{\mathscr{O}_{j}} J_{\mathcal{O}_{i} \cap \mathcal{O}_{j}},
\end{aligned}
$$

namely

$$
J_{\mathscr{O}_{i}} x_{i} J_{\mathscr{O}_{i}}=J_{\mathfrak{O}_{j}} x_{j} J_{\mathcal{O}_{j}}
$$

therefore, it follows by (9.2) that

$$
J_{\mathfrak{O}} y_{i} J_{\mathscr{O}}=J_{\mathscr{O}} y_{j} J_{\mathscr{O}}
$$

6 If there is no vacuum vector the natural restriction maps are the classes of canonical endomorphisms (9.1) modulo inner automorphisms of $\mathscr{A}(\mathcal{O})$ [29] 
and this means that $x \equiv y_{i}$ does not depend on $i$. Now

$$
J_{\mathscr{O}} x J_{\mathscr{O}}=J_{\mathscr{O}} y_{i} J_{\mathscr{O}}=J_{\mathscr{O}_{i}} x_{i} J_{\mathscr{O}_{i}} \in \mathscr{A}\left(\mathcal{O}_{i}\right)^{\prime}
$$

hence

$$
J_{\mathscr{O}} x J_{\mathscr{O}} \in \bigcap \mathscr{A}\left(\mathcal{O}_{i}\right)^{\prime}=\left(\bigvee \mathscr{A}\left(\mathcal{O}_{i}\right)\right)^{\prime}=\mathscr{A}(\mathcal{O})^{\prime}
$$

or

$$
x \in \mathscr{A}(\mathcal{O})
$$

and $\left.x\right|_{O_{i}}=x_{i}$.

Let $\mathscr{A}_{1}, \mathscr{A}_{2}$ be sheaves of von Neumann algebras. A sheaf map $\varrho: \mathscr{A}_{1} \rightarrow \mathscr{A}_{2}$ is a collection of homomorphism $\left\{\varrho_{\mathcal{O}}\right\}$ of $\mathscr{A}_{1}(\mathcal{O})$ into $\mathscr{A}_{2}(\mathcal{O})$ associated to open sets $\mathcal{O} \subset X$ such that

$$
\varrho_{\mathcal{O}} \circ \gamma_{\mathscr{O}, \mathcal{O}}^{(2)}=\gamma_{\hat{\mathcal{O}}, \mathcal{O}}^{(1)} \circ \varrho_{\mathcal{O}},
$$

where the $\gamma^{(i)}$ are the restriction maps of $\mathscr{A}_{i}$. We shall say that $\varrho$ is a sheaf map modulo inners if for $\mathcal{O} \subset \widetilde{\mathcal{O}}$ there exists a unitary $u \in \mathscr{A}_{2}(\mathcal{O})$ (depending on $\mathcal{O}, \widetilde{\mathcal{O}}$ ) such that

$$
\varrho_{0} \circ \gamma_{\hat{0}, \mathcal{O}}^{(2)}=u \gamma_{\tilde{E}, \mathcal{O}}^{(1)} \varrho_{\mathbb{O}}(\cdot) u^{*} \text {. }
$$

One of the implications in the next proposition needs the split and duality assumptions in Sect. 6.

Proposition 9.2. Let $\mathscr{A}$ be a sheaf of von Neumann algebras on the Minkowski space as above and $\varrho$ a morphism localized in a space-like cone $\mathscr{S}$. Then $\varrho$ is translation covariant iff $\varrho$ is a sheaf map modulo inners on the family of the wedge regions $W \supset \mathscr{S}$.

If the vacuum sector is conformally covariant and $\varrho$ is covariant with respect to dilatations, then $\varrho$ is a sheaf map modulo inners with respect to double cones $\mathcal{O}$ as soon as $\varrho$ is localized in $\mathcal{O}$.

Proof. The restriction map associated with an inclusion of wedge regions has the geometrical meaning of a translation (see Sect. 10). If $\varrho$ is translation covariant it follows by Theorem 2.2 and the comments before Lemma 3.2 that $\varrho$ is a sheaf map modulo inners on wedges $W \supset \mathscr{S}$. The converse is true as in Sect. 6, by using the split property for space-like cones and the duality for contractible regions.

The case of a conformally invariant theory is analyzed similarly making use of the geometric interpretation as dilatations of the restriction maps associated with inclusions of double cones [25].

Proposition 9.3. If $\mathscr{A}$ is a sheaf as above and $\varrho$ is localized in a double cone and has finite statistics then $\varrho$ is a sheaf map modulo inners for all double cones $\mathcal{O} \subset \tilde{\mathcal{O}}$ with $\varrho$ localized in $\mathcal{O}$.

Proof. It follows from the analysis in Sect. 2.

\section{Translation Covariance Within the Algebraic Setting}

A crucial tool in this paper was the Bisognano-Wichmann theorem, so far established within the setting of Wightman fields. Borchers [4] has recently obtained a partial converse to this theorem, that will suffice to prove the automatic 
translation covariance of superselection sectors in the Haag-Kastler framework. In this section we assume the vacuum sector to be only translation covariant, with positive energy-momentum; denote by $V$ and $\tau$ the corresponding unitary translation group and action on local algebras. We fix the wedge $W=\left\{\left(a_{0}, \mathbf{a}\right) \in \mathbb{R}^{d} /\left|a_{0}\right|<a_{1}\right\}$, so that by Borchers theorem

$$
J V(a) J=V(-a), \quad a \in \mathbb{R}^{d},
$$

where $J$ is the vacuum modular conjugation of $\mathscr{R}(W)$ and we put $-a \equiv\left(-a_{0},-a_{1}, a_{2}, a_{3}, \ldots\right)$. Let $a$ be a space-like vector with $a_{i}=0, i>1$, so that $W_{-1} \equiv a+W \subset W$. We denote $M=\mathscr{R}(W)$ and $N=\mathscr{R}\left(W_{-1}\right)$. As a corollary one has:

Proposition 10.1. The canonical endomorphism $\gamma: M \rightarrow N$ (with respect to the vacuum) is equal to the action of $\tau_{2 a}$ on $M$.

Proof. We have $J_{N}=V(a) J_{M} V(-a)$, hence

$$
\Gamma=J_{N} J_{M}=V(a) J_{M} V(-a) J_{M}=V(2 a) .
$$

Thus the geometrical interpretation of the canonical endomorphism is purely algebraic.

We now assume that essential duality holds and the net $\mathscr{A}$ is regular in the sense of Sect. 5. We consider an irreducible morphism $\varrho$ localized in a spacelike cone $\mathscr{S} \subset 2 a+W$ and have:

Theorem 10.2. If $\varrho$ has finite statistics, $\varrho$ is translation covariant.

Proof. There exists a DHR conjugate, hence a weak conjugate $\bar{\varrho}$, localized in $W_{-1}$. Let $U$ be a unitary that implements $\varrho$ on $M$ as in the proof of (i) $\Leftrightarrow$ (v) in Theorem 2.2, so that formula (2.7) gives by Proposition 10.1

$$
\operatorname{ad} U(y)=\operatorname{ad}(w) \circ \gamma^{-1} \circ \varrho \circ \gamma(y)=\operatorname{ad}(w) \circ \tau_{-2 a} \circ \varrho \circ \tau_{2 a}(y), \quad y \in M_{1},
$$

for some unitary $w \in M_{1}=\mathscr{R}\left(W_{1}\right)$, with $W_{1}=W-a$.

We now use the fact that $\varrho$ is localized in $W_{-1}$, thus $\operatorname{ad}(\bar{U})=\operatorname{ad}\left(J_{M} U^{*} J_{M}\right)$ acts identically on $\mathscr{R}(\mathcal{O})$ with $\mathcal{O}=W_{-1}^{\prime} \cap W$, therefore, $U \in \mathscr{R}\left(\mathcal{O}_{1}\right)$, where $\mathcal{O}_{1} \equiv-\mathcal{O}$ $=W^{\prime} \cap W_{1}$. Therefore, since $\varrho$ acts identically on $\mathcal{O}_{1}+2 a$, formula (10.1) shows that $w \in \mathscr{R}\left(\mathcal{O}_{1}\right)^{\prime} \cap \mathscr{R}\left(W_{1}\right)$. By the argument in the proof of Theorem 7.1, w normalizes $\mathscr{R}(W)$ and $w$ belongs to $\mathscr{R}(W)$ by the cosimple subfactor argument in Sect. 5. At this point we see that formula (10.1) gives

$$
\operatorname{ad} U(y)=\operatorname{ad}(w) \circ \tau_{-2 a} \circ \varrho \circ \tau_{2 a}(y)
$$

holds not only for all $y \in \mathscr{R}\left(W_{1}\right)$, but also for all $y \in \mathscr{R}\left(W^{\prime}\right)$, hence for all $y$ in the quasi-local $C^{*}$-algebra by additivity, and this proves the theorem as explained in Sect. 3.

\section{A Look at Infinite Statistics}

The analysis of this paper is to a large extent independent of any finite statistics assumption. For a massive theory finite statistics follows automatically [16] and there is no known example of (irreducible) superselection sector with infinite 
statistics. In this section we sketch a construction of the unbounded normal left inverse of a morphism with infinite statistics that indicates that infinite statistics could be associated with physically relevant situations.

Suppose that $\varrho$ is a morphism localized in a wedge region $W$, Poincaré covariant with infinite statistics. We show here how to construct an unbounded, locally normal, left inverse $\phi$ of $\varrho$, indeed, we shall have

$$
\phi=\varrho^{-1} \circ E,
$$

where $E: \mathscr{A} \rightarrow \varrho(\mathscr{A})$ is a locally normal operator valued weight [20]. We construct a normal operator valued weight $E_{W}: M \rightarrow \varrho(M)$, where $M=\mathscr{R}(W)$; Poincaré covariance implies, as in Sect. 3 , that as $W$ varies $E_{W}$ is a consistent family determining $E$.

To construct $E$ recall that the covariance with respect to the boost action $\left\{\alpha_{t}, t \in \mathbb{R}\right\}$ leaving $\mathscr{R}(W)$ stable is equivalent to

$$
\alpha_{t}\left(\varrho\left(\alpha_{-t}(x)\right)\right)=z_{t}^{*} \varrho(x) z_{t}, \quad x \in \mathscr{A},
$$

where $z_{t}$ is a $\alpha$-cocycle that belongs to $\mathscr{R}(W)$ by essential duality. By the BisognanoWichmann theorem $\left.\alpha_{t}\right|_{M}$ is the modular group of $M$ with respect to the vacuum state $\omega_{0}$ (we rescale the boost parameter by $2 \pi$ ) hence, by a theorem of Connes [11], there exists a normal faithful semifinite weight $\varphi$ of $M$ such that

$$
\sigma_{t}^{\varphi}(x)=z_{t} \alpha_{t}(x) z_{t}^{*}, \quad x \in M,
$$

where $\sigma^{\varphi}$ is the corresponding modular group, in fact, $\left(D \varphi: D \omega_{0}\right)_{t}=z_{t}$.

We thus have

$$
\begin{aligned}
\sigma_{t}^{\varphi}(\varrho(x)) & =z_{t} \alpha_{t}(\varrho(x)) z_{t}^{*} \\
& =z_{t}\left(\alpha_{t} \circ \varrho \circ \alpha_{-t}\left(\alpha_{t}(x)\right)\right) z_{t}^{*} \\
& =\varrho\left(\alpha_{t}(x)\right), \quad x \in M,
\end{aligned}
$$

in particular, $\sigma_{t}^{\varphi}$ leaves $\varrho(M)$ globally invariant and a theorem of Haagerup [20] guarantees the existence of the operator valued weight $M$.

It is possible that infrared particles may be described within this formalism, seemingly related to the concept of particle weight [8].

It is a natural problem to check whether conformal models on the circle provide examples of sectors with infinite statistics, cf. for example the models in [9].

\section{Appendix. Induction, Restriction and Covariance}

In this supplement of Sect. 2 we outline an interpretation of the covariance by the notion of induced representation and correspondences. The concept of induced representation was originated by Frobenius in the context of finite groups and was generalized by Wigner and Mackey to general locally compact groups; it was extended to the setting of bimodules by Rieffel; we refer to the book of Fell and Doran [A1] for a general treatment of the subject. A generalization of this notion is natural in the framework of correspondences and was considered in the $I I_{1}$-subfactor theory [A2]. 
Let $M$ be a von Neumann algebra, that we may assume to be properly infinite (after tensoring by a type $I_{\infty}$ factor). We recall that sectors of $M$ and $M-M$ correspondences (modulo unitary equivalence) can be identified by an argument of Connes, see [27].

There are, however, two restriction operations: if $N \subset M$ is a (properly infinite) von Neumann subalgebra and $\varrho \in \operatorname{End}(M)$, then $[\varrho] \in \operatorname{Sect}(M)$ can be restricted to $N$ is a sector (namely one restricts the associated correspondence). Of course, this sector restriction is only defined up to inner automorphisms of $N$. It is given by

$$
\mathbf{R}_{N}^{M}(\varrho)=\left.\gamma \circ \varrho\right|_{N}
$$

where $\gamma: M \rightarrow N$ is a canonical endomorphism. However, if $\varrho \in \operatorname{End}(M)$ and $\varrho(N) \subset N$, then $\varrho$ can be restricted to an endomorphism of $N$ in the usual way; we call this operation map restriction.

If $\eta \in \operatorname{End}(N)$ we may induce $\eta$ to a sector of $M$. The sector induction is given by

$$
\mathbf{I}_{N}^{M}(\eta)=\varrho \circ \gamma
$$

Yet, if possible, we can extend $\eta$ to an endomorphism of $M$ as a map; we refer to this operation as map extension. The comparison of map restriction and extension with sector restriction and induction is the content of the abstract covariance. Indeed, denote by $\operatorname{End}(N, M)$ the endomorphisms of $M$ that map $N$ into $N$; Theorem 2.2 shows that the endomorphisms $\varrho \in \operatorname{End}(N, M)$ that admit a coherent conjugate are the ones such that the following diagram commutes:

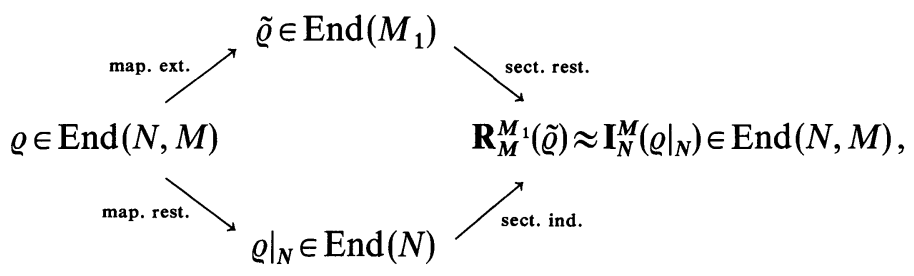

where the map extension $\varrho \in \operatorname{End}\left(M_{1}\right)$ of $\varrho$ is unique as a sector and $\varrho_{1} \approx \varrho_{2}$ in $\operatorname{End}(N, M)$ means that there exists a unitary $u \in N$ with $\varrho_{1}=\operatorname{ad}(u) \circ \varrho_{2}$.

Suppose now $G$ is a locally compact group and $H \subset G$ a closed subgroup, thus $N=V N(H)$ is contained in $M=V N(G)$ (the von Neumann algebra of the left regular representation). Following Connes a representation $\pi$ of $G$ on the Hilbert space $K_{\pi}$ determines a $M-M$ correspondence $\mathscr{H}_{\pi}$ on $L^{2}(G) \otimes K_{\pi}: G$ acts on the left by $l(g) \otimes \pi(g)$ and to the right by $r(g) \otimes$ id $(g \in G)$, where $l$ and $r$ are the left and right regular representations of $G(l$ is quasi-equivalent to $l \otimes \pi)$. Call the associated sector $\Theta_{\pi} \in \operatorname{Sect}(M)$. An analogue statement is, of course, true for $N$ : a representation $\sigma$ of $H$ determines a sector $\Theta_{\sigma} \in \operatorname{Sect}(N)$. If $\sigma=\left.\pi\right|_{H}$ one may show that

$$
\Theta_{\sigma}=\mathbf{R}_{N}^{M}\left(\Theta_{\pi}\right)
$$

and if $\pi$ is the induction of $\sigma$ to $\pi$

$$
\Theta_{\pi}=\mathbf{I}_{N}^{M}\left(\Theta_{\sigma}\right)
$$

Therefore, sector induction and restriction extends the notion of induction and restriction for group representation. 
Suppose now that $N$ is isomorphic to $M$ (this is possible after tensoring by a suitable von Neumann algebra [31]); namely $N=\alpha(M)$ for some $\alpha \in \operatorname{End}(M)$. Then we may identify $M$ with $N$, thus $\operatorname{Sect}(M)$ with $\operatorname{Sect}(N)$. The sector restriction becomes a map $\mathbf{R}: \operatorname{Sect}(M) \rightarrow \operatorname{Sect}(M)$. If $\varrho \in \operatorname{End}(M)$ we have

$$
\mathbf{R}(\varrho)=\bar{\alpha} \circ \varrho \circ \alpha,
$$

in fact, $\mathbf{R}(\varrho)=\left.\gamma \circ \varrho\right|_{N} \sim \alpha^{-1} \circ \gamma \circ \varrho \circ \alpha=\alpha^{-1} \circ \alpha \circ \bar{\alpha} \circ \varrho \circ \alpha=\bar{\alpha} \circ \varrho \circ \alpha$, where $\sim$ denotes the above identification and $\gamma=\alpha \circ \bar{\alpha}$ by [27]. Analogously, the induction is

$$
\mathbf{I}(\varrho)=\alpha \circ \varrho \circ \bar{\alpha},
$$

namely induction and restriction are maps of the same type.

In particular one may show the Frobenius reciprocity theorem, see [A 2] for implicit statements. If $M$ is a factor and $\varrho, \eta \in \operatorname{End}(M)$ are irreducible with finite index, then $\alpha \circ \varrho \circ \bar{\alpha} \succ \eta$ (with multiplicity $n$ ) iff $\bar{\alpha} \circ \eta \circ \alpha \succ \varrho$ (with multiplicity $n$ ), provided that $\alpha$ is a direct sum of sectors with finite index.

\section{References}

1. Araki, H.: Von Neumann algebras of local observables for the free scalar field. J. Math. Phys. 5, 1-13 (1964)

2. Bisognano, J.J., Wichmann, E.: On the duality condition for a hermitean scalar field. J. Math. Phys. 16, 985-1007 (1975)

Bisognano, J.J., Wichmann, E.: On the duality condition for quantum fields. J. Math. Phys. 17, 303-321 (1976)

3. Borchers, H.J.: Local rings and connection of spin and statistics. Commun. Math. Phys. 1, 281-307 (1965)

4. Borchers, H.J.: The CPT theorem in two-dimensional theories of local observables. Commun. Math. Phys. 143, 315 (1992)

5. Bredon, G.E.: Sheaf theory. New York: McGraw-Hill 1967

6. Buchholz, D., Mack, G., Todorov, I.: The current algebra on the circle as a germ of local field theories. Nucl. Phys. B (Proc. Suppl.) 5 B, 20 (1988)

Buchholz, D., Mack, G., Todorov, I.: Localized automorphisms of the U(1)-current algebra: An instructive example. In: The algebraic structure of superselection sectors. Kastler, D. (ed.) Singapore: World Scientific 1990

7. Buchholz, D., Fredenhagen, K.: Locality and structure of particle states. Commun. Math. Phys. 84, 1-54 (1982)

Fredenhagen, K.: Localizability of particle states. In: The algebaic structure of superselection sectors. Kastler, D. (ed.) Singapore: World Scientific 1990

8. Buchholz, D., Pormann, M., Stein, M.: Dirac versus Wigner: Towards a universal particle concept in local quantum field theory. Preprint

9. Buchholz, D., Schulz-Mirbach, H.: Haag duality in conformal quantum field theory. Rev. Math. Phys. 2, 105 (1990)

10. Buchholz, D.: Product states for local algebras. Commun. Math. Phys. 36, 287 (1974) Buchholz, D., Wichmann, E.: Causal independence and the energy-level density of states in quantum field theory. Commun. Math. Phys. 106, 321-344 (1986)

Buchholz, D., D’Antoni, C., Longo, R.: Nuclear maps and modular structures I \& II. J. Funct. Anal. 88, 223-250 (1990); Commun. Math. Phys. 129, 115-138 (1990)

Fidaleo, A.: Matricially nuclear maps. J. Oper. Theory (to appear)

11. Connes, A.: Une classification des facteurs de type III. Ann. Sci. Ec. Norm. Sup. 8, 383-419 (1975) 
12. D'Antoni, C., Longo, R.: Interpolation by type I factors and the flip automorphisms. J. Funct. Anal. 51, 361-371 (1983)

Doplicher, S., Longo, R.: Standard and split inclusions of von Neumann algebras. Invent. Math. 75, 493-536 (1984)

13. D'Antoni, C., Fredenhagen, K.: Charges on space-like cones. Commun. Math. Phys. 94, 537-544 (1984)

14. Doplicher, S., Haag, R., Roberts, J.E.: Local observables and particle statistics. I. Commun. Math. Phys. 23, 199-230 (1971)

15. Doplicher, S., Haag, R., Roberts, J.E.: Local observables and particle statistics. II. Commun. Math. Phys. 35, 49-85 (1974)

Doplicher, S.: The statistics of particles in local quantum theory. In: Lecture Notes in Physics, vol. 39. Berlin, Heidelberg, New York: Springer 1975

16. Fredenhagen, K.: On the existence of antiparticles. Commun. Math. Phys. 79, 141 (1981)

17. Fredenhagen, K.: Generalization of the theory of superselection. In: The algebraic theory of superselection sectors. Kastler, D. (ed.) Singapore: World Scientific 1990

18. Fredenhagen, K., Rehren, K.H., Schroer, B.: Superselection sectors with braid group statistics and exchange algebras, I: General theory. Commun. Math. Phys. 125, 201-226 (1989)

19. Haag, R., Kastler, D.: An algebraic approach to quantum field theory. J. Math. Phys. 5, 848-861 (1964)

20. Haagerup, U.: Operator valued weights in von Neumann algebras. I, II. J. Funct. Anal. 32, 175-206 (1979); 33, 239-261 (1979)

21. Hislop, P., Longo, R.: Modular structure of the von Neumann algebras associated with the free scalar massless field theory. Commun. Math. Phys. 84, 71-85 (1982)

22. Kosaki, H.: Extension of Jones' theory on index to arbitrary factors. J. Funct. Anal. 66, 123-140 (1986)

23. Izumi, M.: Application of fusion rules to classification of subfactors. Publ. RIMS 27, 953-994 (1991)

24. Jones, V.R.F.: Index for subfactors. Invent. Math. 72, 1-25 (1983)

25. Longo, R.: The joint modular structure of an inclusion of von Neumann algebras. Contemp. Math. 62, 529-538 (1987)

26. Longo, R.: Index of subfactors and statistics of quantum fields. I. Commun. Math. Phys. 126, 217-247 (1989)

27. Longo, R.: Index of subfactors and statistics of quantum fields. II: Correspondences, braid group statistics and Jones polynomial. Commun. Math. Phys. 130, 285-309 (1990)

28. Longo, R.: Solution of the factorial Stone-Weierstrass conjecture. Invent. Math. 76, 145-155 (1984)

29. Longo, R.: Simple injective subfactors. Adv. Math. 63, 152-171 (1986)

30. Longo, R.: Algebraic and modular structures of von Neumann algebras of physics. Proc. Sympos. Pure Math. 38, 551-566 (1982)

31. Longo, R.: Minimal index and braided subfactors. J. Funct. Anal. (to appear)

32. Pimsner, M., Popa, S.: Entropy and index for subfactors. Ann. Sci. Ec. Norm. Sup. 19, 57-106 (1986)

33. Roberts, J.E.: New light on the mathematical structure of algebraic field theory. Proc. Sympos. Pure Math. 38, 523-550 (1982)

34. Roberts, J.E.: Local cohomology and superselection structure. Commun. Math. Phys. 51, 107-119 (1976)

Roberts, J.E.: Lectures in algebraic quantum field theory. In: The algebraic structure of superselection sectors. Kastler, D. (ed.) Singapore: World Scientific 1990

35. Streater, R.F., Wightman, A.S.: PCT, spin and statistics, and all that. Reading, MA: Addison-Wesley 1989

36. Takesaki, M.: Conditional expectation in von Neumann algebras. J. Funct. Anal. 3, 306-321 (1970)

37. Takesaki, M.: Tomita's theory of modular Hilbert algebras and its applications. Lecture Notes in Mathematics, vol. 128. Berlin, Heidelberg, New York: Springer 1970

38. Wick, G.C., Wightman, A.S., Wigner, E.P.: The intrinsic parity of elementary particles. Phys. Rev. 88, 101-105 (1952) 
A1. Fell,' I.M.G., Doran, R.S.: Representation of *-algebras, locally compact groups and Banach *-algebraic bundles, vol. 1 \& 2. New York: Academic Press 1988

A2. Connes, A.: Unpublished

Popa, S.: Correspondences. Preprint

Ocneanu, A.: Quantized groups, string algebras and Galois theory for algebras. In: London Math. Soc. Lect. Notes Series, vol. 136, pp. 119-172. Cambridge: Cambridge University Press 1988

Communicated by A. Connes 
